University of Wollongong

Research Online

Faculty of Engineering and Information

Faculty of Engineering and Information

Sciences - Papers: Part A

Sciences

$1-1-2016$

\title{
Transient CFD modelling of low-temperature spontaneous heating behaviour in multiple coal stockpiles with wind forced convection
}

Jian Zhang

University of Wollongong, jz164@uowmail.edu.au

Yuntao Liang

Shenyang Branch of China Coal Research Institute

Ting X. Ren

University of Wollongong, tren@uow.edu.au

Zhongwei Wang

University of Wollongong, zhongwei@uow.edu.au

Gongda Wang

University of Wollongong, Mine Safety Technology Branch of China Coal Research Institute, gongdaw@uow.edu.au

Follow this and additional works at: https://ro.uow.edu.au/eispapers

Part of the Engineering Commons, and the Science and Technology Studies Commons

Research Online is the open access institutional repository for the University of Wollongong. For further information contact the UOW Library: research-pubs@uow.edu.au 


\title{
Transient CFD modelling of low-temperature spontaneous heating behaviour in multiple coal stockpiles with wind forced convection
}

\begin{abstract}
Spontaneous heating of coal stockpile has long been a thermal dynamic hazard during coal storage, processing, and transport. A transient non-equilibrium thermal CFD model has been developed to study the low-temperature self-heating behaviour of coal in multiple stockpiles under different prevailing wind conditions. Modelling results from the initial steady wind flow simulation indicate that a wake region can be induced on the leeward side of each coal stockpile. Pressure coefficient drops when the wind stream encounters or leaves a stockpile and the pressure coefficient profiles of multiple stockpiles tend to have more resemblance with a wider spacing. The first stockpile acts like a wind barrier to the adjacent stockpiles and the maximum temperature of it tends to be the first approaching the critical temperature. A 'hot spot' will develop and then migrate towards deep regions in each of these stockpiles that are loosely compacted under higher wind velocity conditions. Wind velocity and porosity of stockpile have significant influences on self-heating behaviour of the stockpiles and transport pattern of gaseous products liberated by coal oxidation. Compacting stockpiles from loosely packed scenario to slightly packed scenario might not be able to slow down the temperature rising rate at low-temperature range but could considerably minimize the volume of deteriorated coal. The highest temperature rising profiles of the stockpiles located in downwind side can approach to that of the first stockpile, particularly when they are more widely stacked. Stacking coal stockpiles as close as practically possible is recommended to maximise the "protection" of adjacent stockpiles but would cause undesirable accumulation of carbonic gases. Stockpiles in low height and gentle slope will have a prolonged safe storage period, especially for the first stockpile directly facing the wind direction. However, it may not slow down the self-oxidation process of the adjacent stockpile at very initial stage due to "weakened protection" of the first stockpile. This study has practical reference to coal industry especially where multiple coal stockpiles require to be constructed.
\end{abstract}

\section{Keywords}

multiple, behaviour, heating, spontaneous, temperature, low, convection, modelling, cfd, transient, wind, stockpiles, coal, forced

\author{
Disciplines \\ Engineering | Science and Technology Studies
}

\section{Publication Details}

Zhang, J., Liang, Y., Ren, T. X., Wang, Z. \& Wang, G. (2016). Transient CFD modelling of low-temperature spontaneous heating behaviour in multiple coal stockpiles with wind forced convection. Fuel Processing Technology, 149 55-74. 


\section{Transient CFD modelling of low-temperature spontaneous heating behaviour in multiple coal stockpiles with wind forced convection}

${ }^{a}$ School of Civil, Mining \& Environmental Engineering, University of Wollongong, NSW 2522, Australia

${ }^{\mathrm{b}}$ Shenyang Branch of China Coal Research Institute, Shenyang, 110016, China

${ }^{\mathrm{c}}$ Mine Safety Technology Branch of China Coal Research Institute, Beijing, 100083, China

\section{Abstract}

Spontaneous heating of coal stockpile has long been a thermal dynamic hazard during coal storage, processing, and transport. A transient non-equilibrium thermal CFD model has been developed to study the low-temperature self-heating behaviour of coal in multiple stockpiles under different prevailing wind conditions. Modelling results from the initial steady wind flow simulation indicate that a wake region can be induced on the leeward side of each coal stockpile. Pressure coefficient drops when the wind stream encounters or leaves a stockpile and the pressure coefficient profiles of multiple stockpiles tend to have more resemblance with a wider spacing. The first stockpile acts like a wind barrier to the adjacent stockpiles and the maximum temperature of it tends to be the first approaching the critical temperature. A 'hot spot' will develop and then migrate towards deep regions in each of these stockpiles that are loosely compacted under higher wind velocity conditions. Wind velocity and porosity of stockpile have significant influences on self-heating behaviour of the stockpiles and transport pattern of gaseous products liberated by coal oxidation. Compacting stockpiles from loosely packed scenario to slightly packed scenario might not be able to slow down the temperature rising rate at lowtemperature range but could considerably minimise the volume of deteriorated coal. The highest temperature rising profiles of the stockpiles located in downwind side can approach to that of the first stockpile, particularly when they are more widely stacked. Stacking coal stockpiles as close as practically possible is recommended to maximise the "protection" of adjacent stockpiles but would cause undesirable accumulation of carbonic gases. Stockpiles in low height and gentle slope will have a prolonged safe storage period, especially for the first stockpile directly facing the wind direction. However, it may not slow down the self-oxidation process of the adjacent stockpile at very initial stage due to "weakened protection" of the first stockpile. This study has practical reference to coal industry especially where multiple coal stockpiles require to be constructed.

Keywords: Spontaneous heating, low temperature oxidation, Coal stockpile, CFD modelling

\footnotetext{
${ }^{*}$ Corresponding author.

Address: Faculty of Engineering and Information Sciences, University of Wollongong, NSW 2500, Australia Tel.: +61 432806 678. E-mail addresses: jz164@ uowmail.edu.au (Jian Zhang).
} 


\begin{tabular}{|c|c|c|}
\hline$C_{\mathrm{p}}$ & Specific heat capacity & $\mathrm{J} \mathrm{kg}^{-1} \mathrm{~K}^{-1}$ \\
\hline$D$ & Mass diffusion coefficient of specie in gas mixture & $\mathrm{kg} \mathrm{m}^{2} \mathrm{~s}^{-1}$ \\
\hline$D_{T}$ & Thermal diffusion coefficient of specie in gas mixture & $\mathrm{kg} \mathrm{m}^{2} \mathrm{~s}^{-1}$ \\
\hline$E_{\mathrm{a}}$ & Apparent activation energy & $\mathrm{kJ} \mathrm{mol}^{-1}$ \\
\hline$\Delta H$ & Heat of coal oxidation & $\mathrm{kJ} \mathrm{mol}^{-1} \mathrm{O}_{2}$ \\
\hline $\mathrm{n}$ & Apparent order of reaction & \\
\hline$A$ & Arrhenius pre-exponential factor & $\left(\mathrm{kmol} / \mathrm{m}^{3}\right)^{1-\mathrm{n}} \mathrm{s}^{-1}$ \\
\hline$R$ & Universe gas constant & $\mathrm{kJ} \mathrm{mol}^{-1} \mathrm{~K}$ \\
\hline r & Consumption rate of oxygen & $\mathrm{kmol} \mathrm{m}^{-3} \mathrm{~s}^{-1}$ \\
\hline$T$ & Temperature & $\mathrm{K}$ \\
\hline $\mathrm{t}$ & Time & $\mathrm{s}$ \\
\hline$\vec{v}$ & Gas flow velocity vector & \\
\hline C & Gas species concentration & $\mathrm{kmol} \mathrm{m}^{-3}$ \\
\hline$P$ & Pressure & $\mathrm{Pa}$ \\
\hline $\mathrm{k}$ & Permeability of the coal matrix & $\mathrm{m}^{2}$ \\
\hline $\mathrm{g}$ & Gravitational acceleration & $\mathrm{m} \mathrm{s}^{-2}$ \\
\hline $\mathrm{d}$ & Diameter of coal particle & $\mathrm{m}$ \\
\hline V & Wind velocity & $\mathrm{m} \mathrm{s}^{-1}$ \\
\hline$h$ & Heat transfer coefficient & $\mathrm{W} \mathrm{m}{ }^{-2} \mathrm{~K}^{-1}$ \\
\hline$\vec{F}$ & Body force vector & $\mathrm{N} \mathrm{m}^{-3}$ \\
\hline $\mathrm{H}$ & Height & $\mathrm{m}$ \\
\hline$(\mathrm{x}, \mathrm{y})$ & Cartesian coordinates & $\mathrm{m}$ \\
\hline \multicolumn{3}{|c|}{ Greek letters } \\
\hline$\rho$ & Density & $\mathrm{kg} \mathrm{m}^{-3}$ \\
\hline$\varepsilon$ & Porosity & \\
\hline$\lambda$ & Thermal conductivity & $\mathrm{W} \mathrm{m}{ }^{-1} \mathrm{~K}^{-1}$ \\
\hline$\mu$ & Gas viscosity & $\mathrm{kg} \mathrm{m} \mathrm{s}^{-2}$ \\
\hline$\vec{\tau}$ & Viscous stress tensor & $\mathrm{N} \mathrm{m}^{-2}$ \\
\hline \multicolumn{3}{|c|}{ Subscripts } \\
\hline g & Gas & \\
\hline c & Coal & \\
\hline $\mathrm{a}$ & Ambient & \\
\hline o & Oxygen & \\
\hline $\mathrm{i}$ & Carbon dioxide & \\
\hline $\mathrm{j}$ & Carbon monoxide & \\
\hline $\mathrm{R}$ & Reference & \\
\hline$\varphi$ & Pressure coefficient & \\
\hline
\end{tabular}


37 A number of large open cut coal mining operations in Queensland of Australia are expected to commence in the near future. High volume of coal requires temporarily stored as one or multiple stockpiles in a storage yard of a coal mine site or a port before they can be transported to other destinations. Coal, as a carbonaceous material, is able to be oxidised and to liberate heat at ambient temperature with the presence of oxygen rich air [1-4]. Self-heating of coal stockpile will occur if the heat generated by coal oxidation is not adequately dissipated. The accumulated heat will result in a slow rising of temperature at the initial stage of coal oxidation. Once the temperature reaches a critical value which was widely reported to be $60 \sim 120^{\circ} \mathrm{C}$, thermal runaway would occur and the self-heating rate of coal is very likely to take off in a relatively short period of time [5-11]. Therefore low-temperature coal oxidation is a very critical stage with concern of preventing coal spontaneous combustion. Self-heating of coal in stockpiles has long been regarded as a safety concern and additionally, pre-oxidation of coal would incur considerable loss of coal calorific value and seriously affect caking property [12-14]. More recently the liberation of large amounts of greenhouse-relevant gases and hazardous substances, such as arsenic, mercury, and lead from coal stockpile combustion or oxidation has raised considerable concerns from global communities [15-18]. Despite the long history of the problem, it has also been accepted that the physical and chemical processes responsible for this problem is extremely complex [12]. Derivation of an analytical solution to the problem would be a formidable or even impossible task and consequently, the problem is often addressed numerically. Prior to the availability of high performance fluid-thermodynamics computation code, several preliminary mathematical models have been developed to investigate the issue numerically [19-33]. These studies provided valuable insights into this problem but the accuracy and applicability is questioned because these models were derived with many simplifications and limitations.

To date with the advance of Computational Fluid Dynamics (CFD) modelling techniques, predictable interpretation of self-heating of piled carbonaceous material within reasonable engineering accuracy becomes more approachable $[9,14$, 34-41]. Among these works, Moghtaderi et al. [34]studied the effects of wind on self-heating behaviour of coal stockpile and found forced convection plays a critical role in dynamics of flow field inside the stockpile and thereby affects the self-heating process. Yuan and Smith studied effects of coal properties on self-heating problem in underground coal mine goaf areas by a CFD model and later on developed a 3D equilibrium thermal model to simulate spontaneous heating in a large-scale coal chamber with a forced ventilation system [35, 36]. Ejlali et al. [37] improved preceding models by employing a non-equilibrium thermal approach and found the maximum temperature is a function of porosity of porous 
advance rate on spontaneous heating in goaf areas by a CFD model. Kim and Sohn [40] numerically validated a novel method to suppress spontaneous ignition of coal stockpiles in a coal storage yard. Zhu et al. [9] proposed a theoretical model to predict the incubation period and location of hot spot of a coarse coal stockpile. Taraba et al. [39] modelled real fluctuations of the wind flow and observed a movement of hot spot if full buoyancy effect was activated. Yang et al. [14] proposed a two-dimensional model to investigate the effects of coating the bottom of coal stockpile with fine particles to control self-heating hazard. Xia et al. [41] also used the CFD code to simulate the self-heating process of residual coal in longwall goaf. In addition considerable parametric studies have been conducted by these models and a number of practical suggestions were delivered to coal industry. Numerical solutions to self-heating of coal stockpile usually only deal with a single coal stockpile scenario but practically, multiple stockpiles are probably required to be stacked in storage yard of a coal mine or a port to increase storage capacity. Coal stockpiles stacked in these places may be subject to long period of exposure of wind flow which has been demonstrated to have significant influences on self-heating behaviour of stockpiled coal $[9,34,39]$. Therefore self-heating behavior of multiple stockpiles which are packed in proximity requires to be studied especially at low temperature range with prevailing wind conditions. The purpose of this work is to investigate the low-temperature self-heating characteristics of multiple coal stockpiles under wind flow condition and with validation, to perform variable analysis to gain more understandings of the problem in multi-stockpile scenario.

\section{$82 \quad 2$ Brief mechanism of spontaneous heating of coal stockpile}

A coal stockpile is essentially a porous medium consisting of heterogeneous coal particles and the voids between coal pellets are filled with mixture of fresh air and gaseous products liberated by coal oxidation. Interaction of coal with oxygen including coal oxidation and oxygen adsorption at low temperature is exothermic as a whole although it could be endothermic at some steps $[1,42]$. Several studies demonstrated that the interaction of coal with water like wetting can also generate heat and it reaches to a general consensus that moisture exhibits either promoting or inhibiting effects on coal oxidation rate but fundamental mechanism especially to a kinetic sense is scarce $[1,43-46]$. As chemical effects of moisture were not understood completely, a few numerical models were developed to investigate only physical effect of moisture on self-heating of coal stockpile and it was indicated interaction of coal with moisture is an efficient heat transfer mechanism in which the vaporisation and diffusion of water from a hot region, followed by condensation in a cooler region, is accompanied by a considerably higher effective rate of heat transfer than that which can occur by conduction alone $[21,26,37,47]$. The efficient heat transfer mechanism was also termed as "heat pipe" effect in some literatures and the "heat pipe" effect usually manifested itself by leading to a levelling of temperature $\left(80 \sim 90^{\circ} \mathrm{C}\right)$ in a coal 
coal and hence it is safe to argue that the major heat generation mechanism responsible for self-heating is coal oxidation

97 [12]. Therefore it is plausible to ignore effects of moisture within temperature ranges below the levelling temperature exerted by "heat pipe" effect. The generated heat is transported into and out of the stockpile by conduction, convection, and radiation. If the rate of heat generation is greater than the rate at which heat can be dissipated in the external environment, it will lead to localised temperature rise and even smouldering/open flame for a long run. Heat conduction occurs between gas-to-gas, gas-to-coal, and coal-to-coal. The low thermal conductivity of coal is the main reason why thermal energy can be well contained in the deep stockpile and cause temperature rising. Heat convection occurs between coal to gas and the efficiency of heat convection is mainly determined by velocity of gas advection within inter-particle channels. Whilst heat radiation could be a major contributor in the surface of stockpile because of solar energy it absorbed but in deep stockpile heat radiation can be negligible [48].

The air stream inside of stockpile can be constantly replaced through wind advection and diffusion caused by oxygen consumption of coal because oxygen consumption reduced the concentration resulting in diffusion if reaction rate is not the same everywhere. Convective movement can be caused by both natural convection and forced convection due to presence of pressure gradient and it has been indicated airflow driven be pressure gradient is a major transport mechanism in porous coal [49]. Diffusive movement is also capable of dispersing gas molecules in or out of coal intraparticle pores. Irrespective of complex pore structure of coal, this study adopts a global surface reaction mechanism so gas transport mechanism in micro-structures like gas diffusion into inner pores is not considered. Besides the main features described above, self-heating process of coal stockpile is also affected by exterior conditions through wind, rain, snow, ambient temperature and solar radiation [12]. Due to the extremely irregularity of meteorological conditions, effects of extrinsic factors except for wind flow are not scope of this study.

\section{The mathematical model}

On basis of the mechanistic understanding of self-heating of coal stockpile, the core constituents of the mathematical model should include description of low-temperature coal oxidation kinetics, energy conservation, species transport, momentum balance and continuity equations for fluid flow in porous medium. For the simplicity of the solution, terms of heat radiation and effect of moisture are not included in the model.

\subsection{Low-temperature coal oxidation kinetics}

The most intriguing puzzle would be the low-temperature interaction of coal with oxygen because of complex nature of coal. Despite of the mechanism of coal oxidation having not been completely solved, briefly two parallel interaction sequences were identified: one is direct burn-off and another one is adsorption sequence [1]. Solid and gaseous products 
are also concomitant during the two sequences and details of them are omitted here. To define coal-oxygen reaction rate with elevated temperature to a mathematical sense, a simple finite rate Arrhenius reaction mechanism is often used $[9,35$, $36,38,39,41]$, which is given,

$$
r=A\left[C_{\mathrm{o}}\right]^{n} \exp \left(-E_{\mathrm{a}} / R T\right)
$$

It is assumed that oxygen can penetrate throughout the coal without diffusional limitation and the reaction mechanism between gas and solid phases is deemed as a homogenous gas phase reaction [11]. The value of the reaction order in low temperature oxidation of coal and other carbonaceous materials has been indicated to vary from 0.5 to $1[12,47]$. This model approximates first order of coal oxidation. It has also been reported that the value of apparent activation energy of different coals can vary between 12 and $95 \mathrm{~kJ} / \mathrm{mol}$ [36] and more specific values of activation energy for low temperature coal oxidation can be found in the review work conducted by Wang and co-workers [1]. Taraba and Michalec [38] discussed the pre-exponential factor is of the greatest uncertainty giving ordinarily values differing over several orders and another study also indicated the pre-exponential factor has a typical value between 1 and $7 \times 10^{5} / \mathrm{s}$ [35]. It can be also seen the rate of coal oxidation is not only affected by the Arrhenius constants but also by partial pressure of oxygen at a given reaction order and temperature. Therefore any solid coal-oxygen complex produced during low-temperature coal oxidation would not affect the rate of oxidation if Arrhenius-rate reaction mechanism is assumed. Based on experimental data, Yuan and Smith $[35,36]$ generalised that consuming one mole of oxygen by coal would generate one mole carbon dioxide and roughly 0.1 mole carbon monoxide plus heat at the early stage of coal oxidation, which yields a very simplified stoichiometric scheme of low-temperature coal oxidation:

$$
\text { Coal }+\mathrm{O}_{2} \rightarrow \mathrm{CO}_{2}+0.1 \mathrm{CO}+\text { Heat }
$$

145 This model also employs this coal oxidation reaction scheme at low temperature range. Kaji et al. [2] measured the rates 146 of heat liberation and oxygen consumption due to coal oxidation at low temperature range using coals ranging from 147 subbituminous to anthracite and 300 379 kJ heat evolved per mole of oxygen at steady state was reported. Many works $148[23,28,29,36,41]$ used $300 \mathrm{~kJ} / \mathrm{mol}$ oxygen as coal oxidation reaction heat so this model also uses such a value as reaction heat at low temperature range.

\section{$150 \quad 3.2$ Energy conservation}

151 Normally heat of chemical reaction is liberated due to variation of enthalpy between reactants and products. It is usually 152 the case when modeling extremely drastic reaction like fuel combustion. Coal oxidation rate at low-temperature is however very slow and therefore the heat generated by coal oxidation is modeled as a source term and written as a User

154 Defined Function (UDF). To be more realistic to describe heat interaction inside coal stockpile, heat transfer between coal particles should be treated in a non-equilibrium approach. The temperature differential between the solid coal 
particle and gas stream is important in the thermal behaviour and it is thus necessary to represent the energy stored in each individual phase as well as the exchange of thermal energy between them, which gives energy conservation for solid coal pellet:

$$
(1-\varepsilon) \rho_{\mathrm{c}} C_{\mathrm{pc}} \frac{\partial T_{\mathrm{c}}}{\partial t}=(1-\varepsilon) \lambda_{\mathrm{c}} \nabla^{2} T_{\mathrm{c}}+\frac{6(1-\varepsilon)}{d \varepsilon} h\left(T_{\mathrm{g}}-T_{\mathrm{c}}\right)+r \Delta H
$$

In which the successive terms represent internal energy growth of coal particle, heat diffusion in solid coal, heat convection interacted with gas stream, and heat generated by coal oxidation which is a source term. In view of temperature variation is not significant in low-temperature self-heating of coal stockpile and to produce a faster convergence, many models assumed the validity of the Boussinesq approximation $[9,21,25-27,30]$. This approximation essentially states that the temperature variation of the fluid properties can be ignored except for the density, and that the density dependence is only considered when it gives rise to buoyancy convection in natural convection driven flows [12]. However Boussinesq approximation is not suggested to be used with species calculation and reacting flow involved otherwise accuracy of result is very likely to become inacceptable [50]. Therefore ideal compressible gas flow is considered and energy balance for the gas stream is written as:

$$
\varepsilon \frac{\partial}{\partial t}\left(T_{\mathrm{g}} \rho_{\mathrm{g}} C_{\mathrm{pg}}\right)+\nabla\left[\vec{v}\left(T_{\mathrm{g}} \rho_{\mathrm{g}} C_{\mathrm{pg}}+P\right)\right]=\varepsilon \lambda_{\mathrm{g}} \nabla^{2} T_{\mathrm{g}}-\frac{6(1-\varepsilon)}{d \varepsilon} h\left(T_{\mathrm{g}}-T_{\mathrm{c}}\right)
$$

170 In which the successive terms represent transient energy rise of gas stream, heat convection of gas stream, heat diffusion 171 in gas stream, and heat convection interacted with solid coal.

\section{$172 \quad 3.3$ Species conservation}

173 Nitrogen is neither consumed nor produced during whole process of self-heating so species conservation is mainly 174 focused on oxygen, carbon dioxide, and carbon monoxide in gas stream according to the assumed reaction scheme, which give rise to the species conservation:

$$
\left\{\begin{array}{c}
\text { For oxygen: } \varepsilon \frac{\partial\left(\rho C_{\mathrm{o}}\right)}{\partial t}+\varepsilon \nabla\left(\rho \vec{v} C_{\mathrm{o}}\right)-\nabla\left(\rho D_{\mathrm{o}} \nabla C_{\mathrm{o}}+D_{\mathrm{T}, \mathrm{o}} \frac{\nabla T}{T}\right)+r=0 \\
\text { For carbon dioxide: } \varepsilon \frac{\partial\left(\rho C_{\mathrm{i}}\right)}{\partial t}+\varepsilon \nabla\left(\rho \vec{v} C_{\mathrm{i}}\right)-\nabla\left(\rho D_{\mathrm{i}} \nabla C_{\mathrm{i}}+D_{\mathrm{T}, \mathrm{i}} \frac{\nabla T}{T}\right)-r=0 \\
\text { For carbon monoxide: } \varepsilon \frac{\partial\left(\rho C_{\mathrm{j}}\right)}{\partial t}+\varepsilon \nabla\left(\rho \vec{v} C_{\mathrm{j}}\right)-\nabla\left(\rho D_{\mathrm{j}} \nabla C_{\mathrm{j}}+D_{\mathrm{T}, \mathrm{j}} \frac{\nabla T}{T}\right)-0.1 r=0
\end{array}\right.
$$

177 In which the successive terms represent the local accumulation of species, the convective transport of species, the 178 diffusion term of species caused by variation of species concentration and temperature, and the fraction consumed or produced by coal oxidation. 
Navier-Stokes equation is normally used to describe momentum balance for compressible flow as density of fluid varies

with temperature and elapsing of time in this case. Revise Navier-Stokes equation by adding viscous term (last term in

Equation 6) to solve flow momentum balance in porous stockpile, which produces:

$$
\frac{\partial}{\partial t}\left(\rho_{\mathrm{g}} \vec{v}\right)+\nabla\left(\rho_{\mathrm{g}} \vec{v} \vec{v}\right)=-\nabla P+\nabla(\vec{\tau})+\vec{F}-\varepsilon \frac{\mu}{k} \vec{v}
$$

The permeability $k$ of coal matrix is approximated by the Carmen-Kozeny equation for laminar flow in packed beds [51]:

$$
k=\frac{\varepsilon^{3} d^{2}}{150(1-\varepsilon)^{2}}
$$

\subsection{Continuity in porous medium}

Mass change of bulk coal due to coal oxidation at low temperature is ignored. This model also assumes isotropic porous medium and therefore for single phase flow in isotropic porous medium, the continuity equation can be written as:

$$
\frac{\partial \rho}{\partial t}+\nabla(\rho \vec{v})=0
$$

\section{Numerical modelling and validation}

\subsection{Numerical model}

The geometry of the stockpile is considered to be a truncated pyramid. Three identical stockpiles, namely A, B, C, are constructed with a specific height $\&$ side slope and they are located within a certain distance. The length of the stockpile is $40 \mathrm{~m}$. The effects of wind velocity, stockpile height, side slope, porosity, and spacing will be comparatively studied. A base model is selected with details: $4 \mathrm{~m} / \mathrm{s}$ wind velocity, $10 \mathrm{~m}$ high, $50^{\circ}$ side slope, 0.2 porosity and $20 \mathrm{~m}$ spacing. The model consists of two domains: (i) coal stockpile is treated as porous domain and, (ii) the remaining domain is considered as a farfield representing the open space around the stockpiles. The upper and lower boundaries are deemed as adiabatic and no-slip walls. To minimize the limitation of upper wall and outlet boundaries on the flow field around the stockpile, the farfield domain must be sufficiently large. Based on the rule of thumb of large model simulation, the dimension of the farfield domain is decided to be 50m high and 400m long, refer Fig. 1. The geometries of the two domains and mesh of the model is generated via ANSYS 15.0 workbench design modeler and mesh tool, respectively. To utilize non-equilibrium thermal model in porous zone, mesh must be created in very high standard. When activate this model, a fake solid zone which overlaps the porous fluid zone is created, and this solid zone only thermally interacts with the fluid. The discretized mesh generated for the base model via the described method is shown in Fig. 2. Quadrilateral mesh with $1 \mathrm{~m}$ global sizing is used to fill farfield region as show in Fig. 2 (a). As can be observed in Fig. 2 (b), down to near surface of stockpile, 40 thin layers (each layer is $0.1 \mathrm{~m}$ thick) are inflated because this area is expected to be the preferable location of self-heating. Further deep into stockpile $0.5 \mathrm{~m}$ local sizing trilateral mesh is used to fill irregular 
region to meet high standard mesh requirement, otherwise connectivity between fluid zone and fake solid zone will result in immediate error once non-equilibrium thermal model is activated.

Inlet wind profile is modelled as wind power law (refer Equation 9), which essentially states wind velocity progressively approaches to zero at ground surface. Wind velocity of any height can be determined via wind velocity at a reference height. In this study reference height is $10 \mathrm{~m}$ and wind velocity refers to the wind velocity at reference height. Wind velocity profile of the farfield of the base model is shown in Fig. 3, from which it can be observed that wind velocity at inlet varies with the proposed power law.

$$
V=V_{\mathrm{R}}\left(\frac{H}{H_{\mathrm{R}}}\right)^{1 / 7}
$$

218 Wind stream is considered to be fully turbulent in farfield and thus is solved by Fluent RNG k- $\varepsilon$ model. To suppress turbulent viscosity in porous stockpile, laminar zone option is enabled. Many works $[9,22,23,25-27,30,36,37,40]$ have also suggested natural convection is also a possible mechanism of oxygen transport so full buoyancy effect is also activated in this model. Like previous works conducted by Yuan and Smith $[35,36]$, two-step simulation approach is used: (i) a simulation is conducted firstly without turning on chemical reaction and heat source to obtain a steady flow in the farfield and stockpiles; (ii) the transient simulations with surface wall reaction and heat source are conducted using the steady flow field as the initial conditions. As substantial coal adiabatic testing data indicated the temperature of coal would take off after $70^{\circ} \mathrm{C}[6-8]$ and therefore $363 \mathrm{~K}$ is considered as the critical temperature because ambient temperature is assumed as $293 \mathrm{~K}$ in this study. The simulation terminates once maximum temperature of any of the three stockpiles reaches $363 \mathrm{~K}$ or alternatively, it stops running till 90 days to save computation load. Important input parameters of the model are listed as Tab. 1 in which low-temperature coal oxidation kinetic parameters are derived from Taraba's model [39]. The initial and boundary conditions for the model are also tabulated in Tab. 2 in which it should be noted that the initial conditions are listed in terms of steady flow field.

\subsection{Model validation}

This model is validated using published data $[34,36,39]$. To validate the model in more details, an additional model with similar geometric parameters to Taraba's model [39] is established; refer Fig. 4(a). Moghtaderi et al. [34] and Taraba et $a l$. [39] both reported that a wake region was induced right after wind passes a stockpile. This model also identifies this wake region. In addition they also suggested the greatest pressure is exerted on the front face of the pile (windward side) with a marked drop in pressure at the top of the pile before leveling off. This quantity of pressure drop is presented in non-dimensional term using the concept of a pressure coefficient [52]. The pressure coefficient, $\varphi$, is defined via 


$$
\varphi=\frac{2\left(P-P_{\mathrm{R}}\right)}{\rho_{\mathrm{a}} V^{2}}
$$

239 Fig. 4(a) shows the distribution of the pressure coefficient around the validation stockpile at $4 \mathrm{~m} / \mathrm{s}$ wind velocity. As can 240 be seen, the shape of the pressure coefficient curve of this model is very similar to those reported by Moghtaderi et al. 241 [34] and Taraba et al. [39] and the characteristic movement of interior vectors also resembles Taraba's model. Hence it is 242 possible to be deemed that flow field produced by the proposed model is viable.

244 To examine validity of transient temperature rising of the model, an appropriate time step must be selected. Yuan and 245 Smith [36] used one minute time step to simulate self-heating of coalbed and the result was validated by real 246 experimental data in a testing chamber. Based on the previous experience, various time steps (30 seconds, one minute, two minutes, five minutes, and one hour) are studied in this model. It is reconfirmed one minute time step would produce a rational result without incurring too much computation load. In addition various meshing sizes are studied and the mesh independence check suggests that the proposed mesh setting is able to produce a rational result. Slightly more than two thousand iterations are required to reach a convergence of the first-step steady flow field. For the subsequent transient calculation, one thousand iterations were conducted for each time step to render less residual errors for next time step and after running a few initial steps the result reaches convergence for all the remaining time steps. Fig. 5 shows the comparison of maximum temperature rising profile between Taraba's model and the validation model at low-temperature range. It is found the two models report alike and almost linear maximum temperature rising rate at low temperature range. The validation model indicates a slightly slower temperature rising rate and the difference might attribute to the non-equilibrium thermal approach and slightly different model setting. In this regard the model is considered to be robust and viable in simulating both steady wind flow field and unsteady heat transfer field.

\section{$258 \quad 5$ Results and parametric analysis}

$259 \quad 5.1$ Base model result

260 Fig. 3 has reported the wind velocity contour of farfield domain of the base model. It can be seen velocity builds up as wind encounters the first stockpile and then significantly drops after wind passes each stockpile especially at the regions between two stockpiles. It is suspected stockpile A will have the largest quantity of interior airflow than that of the other two stockpiles due to the direct confrontation to wind of stockpile A and a wake region will be induced at the leeward of each stockpile. Fig. 6 illustrates the wind stream vector in both farfield and stockpiles. Three wake regions, namely wake region 1,2, and 3, at the leeward of each stockpile are identified and it appears they are becoming increasingly confined. 
normally signifies more availability of airflow inside stockpile and it can thus be postulated the order of maximum temperature rising rate will follows the same order. To examine airflow travel path inside coal stockpile more fundamentally, interior pressure distribution must be well studied because airflow inside stockpile is greatly driven by pressure gradients. Fig. 8 illustrates gauge pressure distribution and velocity vectors inside of the stockpiles and main air

272 flow travel paths (dash black line) are also sketched based on the pressure gradients. In stockpile A (Fig. 8 (a)), a very low pressure zone is induced at upper-left corner of the stockpile and the largest pressure exerts at the bottom of windward side. Therefore most of the air would travel along path 1 and a hot spot is more readily to evolve along this path due to the significant pressure gradient. Air can also migrate to stockpile along path $2 \mathrm{a}$ and $2 \mathrm{~b}$ due to back flow induced by wake region 1 . The low pressure zone is also able to suck air from top surface of the stockpile, which created airflow travel path 3 . Hot spot hardly evolves along path $2 \mathrm{a}$ due to long travel distance but coal locates at path 3 and $2 \mathrm{~b}$ can be seriously oxidised because of short travel distance. Interestingly a converged airflow is created where airflow along path 1 encounters airflow along path $2 \mathrm{a}$ and the phenomena was termed as "chimney" effect which was reported to be ascribed to natural convection [23] but in this case it appears the phenomena is mainly generated by forced convection. In stockpile B (Fig. 8 (b)), two slightly low pressure zones (zone A and zone B) are induced at both upper corners of the stockpile. The easiest flow path is path 1a due to more appreciable pressure gradient and shorter travelling distance and therefore hot zone is expected to develop along this path. Likewise weak airflow can transport along path 2a while moderate airflow can permeate along path 3 and $2 \mathrm{~b}$ and coal located at these zones is likely to be deteriorated. The "chimney" effect airflow is likely to locate at centre of the stockpile due to more balanced pressure gradients present at both sides. In stockpile C (Fig. 8 (c)), the airflow behaviour resembles that of stockpile B except that the "chimney" airflow moves towards to leeward side because more appreciable pressure gradients exerted at windward side. It is postulated that location of hot spot and deteriorated coal of stockpile C is similar to that of stockpile B.

Fig. 9 depicts the progressive consumption of oxygen of the three stockpiles of the base model at 5, 10, 15, and 20 days respectively. There is very low concentration of oxygen in deep region of stockpiles due to the high resistance of oxygen ingress. The high oxygen concentration zone of stockpile A locates at windward side because of direct confrontation to wind. For stockpiles B and C, high oxygen ingress region favours the two upper corners which are also the low pressure zones from Fig. 8. It is postulated self-heating hazard would develop at these high oxygen concentration zone. As can be seen from Fig. 10, the self-heating favourable zones of the three stockpiles indeed all locates at high oxygen concentration zone and the volume of coal being oxidised of stockpile A is much larger than the other two stockpiles. The maximum temperature rising rates of the three stockpiles are also plotted, refer Fig. 11 in which the overall trend of 
temperature rising rate, as predicted, is $\mathrm{A}>\mathrm{C}>\mathrm{B}$ but the rising rate of stockpile $\mathrm{C}$ overlaps with that of stockpile $\mathrm{B}$ after 40

days as a possible result of oxygen depletion. To further examine the transport pattern of gaseous product liberated from coal oxidation inside the stockpiles, the contours of $\mathrm{CO}_{2}$ are also captured, refer Fig. 12. It is noticeable high concentration of this carbonic gas is retained in the interior of the stockpiles. A possible explanation is the production rate of the gaseous product outweighs its dissipation rate due to weak airflow inside of stockpiles. Additionally it can be found the highest accumulation of $\mathrm{CO}_{2}$ develops at leeward side for stockpile A due to the insufficient dilution of weak backflow, while at windward side and beneath top for stockpile $\mathrm{B}$ and $\mathrm{C}$, which implies the air leakage along path $1 \mathrm{~b}$ and 3 (Fig. 8-b, c) is not sufficiently strong to disperse the high concentration of $\mathrm{CO}_{2}$ and the "chimney" effect would induce a high concentration of $\mathrm{CO}_{2}$ zone beneath the top of stockpiles. Considering the emission of greenhouse effect gases contributed from low-temperature oxidation and spontaneous combustion of coal stockpile has been recently regarded as a highly concerned issue [16], the characteristic flow pattern of $\mathrm{CO}_{2}$ dispersion and transport at exterior of stockpiles is also investigated, refer Fig. 13. It is not hard to observe that more $\mathrm{CO}_{2}$ is generated as self-heating of coal stockpile advances and $\mathrm{CO}_{2}$ tends to accumulate at regions between two stockpiles. The high concentration of carbonic gases may raise another safety issue if coal operators were under exposure of such concentrated carbonic gas for a long period of time.

\subsection{Parametric study}

\subsubsection{Wind velocity}

Fig. 14 shows temperature contours of the three stockpiles after 30 days at different wind velocities and Fig. 15 shows maximum temperature rising profiles of the three stockpiles at various wind velocities. It can be seen wind velocity has significant influences on spontaneous heating process and volume of coal being seriously oxidised of the three stockpiles especially stockpile A. Both maximum temperature rising rate and volume of deteriorated coal considerably increase at a higher wind velocity. For example, if wind velocity is as low as $2 \mathrm{~m} / \mathrm{s}$, maximum temperatures of the three stockpiles are not able to reach critical value in 90 days. For the highest wind velocity $6 \mathrm{~m} / \mathrm{s}$, maximum temperature of stockpile A reaches $363 \mathrm{~K}$ in one month; whilst for medium wind velocity $4 \mathrm{~m} / \mathrm{s}$, the incubation period prior to the critical temperature for stockpile A is 48 days. As previously discussed stockpile A stands like a wind barrier to stockpiles behind it, it is not surprised to notice maximum temperature rise rate of stockpile B and C is slower than that of stockpile A even at various wind velocities. In practical, wind barrier has been proposed as a possible control to coal stockpile spontaneous combustion hazard [53]. The coal oxidation at low wind velocity might give off less amount of carbonic gas but it would be more difficult to dilute the gases product, refer Fig. 16. It can be found the increased wind velocity can disperse the generated $\mathrm{CO}_{2}$ more effectively and high wind velocity therefore might be a favourable parameter in this regard. 


\subsubsection{Spacing}

329 As stockpile A is essentially a wind barrier to stockpile B and C, if stockpiles stacked behind stockpile A are located within wake region of stockpile $\mathrm{A}$, it is postulated the maximum temperature rising rate will always lag behind stockpile A. Flow field for a single stockpile is performed and it is found the wake region is approximately $70 \mathrm{~m}$ in length as wind velocity is $4 \mathrm{~m} / \mathrm{s}$, side slope is $50^{\circ}$, and height of stockpile is $10 \mathrm{~m}$. Therefore spacing $5 \mathrm{~m}, 20 \mathrm{~m}$, and $40 \mathrm{~m}$ are chosen to examine effects of spacing within wake region and $80 \mathrm{~m}$ is selected to compare wide spacing out of wake region. To acquire more understandings of effects of different positions of coal stockpiles, pressure profile around them must be well studied because pressure drop is the main driver of the airflow advection. The pressure coefficient profiles of different spacing of stockpiles are plotted, refer Fig. 4 and Fig. 17. It is indicated that different spacing almost have no effects on pressure coefficient distribution of stockpile A and it is plausible to predict that the self-heating behaviour of stockpile A will remain unchanged. It is also found pressure drops increasingly steeper with wider spacing when wind encounters stockpile B and C, which implies more airflow would be introduced to the stockpiles and more heat would be generated. Fig. 18 shows the maximum temperature rising rates of the three stockpiles with various spacing. It is obvious spacing has little influence on stockpile A and maximum temperature develops more rapidly of stockpile B and C with wider spacing due to larger pressure variation. The maximum temperature rising profiles of stockpile $\mathrm{B}$ and $\mathrm{C}$ gradually approach to stockpile A with increasingly wider spacing. It appears stockpiles B and C lose most of the "protection" of stockpile A with wider spacing. Inversely, maximum temperature rising profiles of stockpile B and C gradually separate with stockpile A as geometrically approach to stockpile A. Fig. 19 shows temperature contour of stockpile B with different spacing at 30 day. It is quite clear the hot spot zone $(350 \mathrm{~K})$ is gradually enlarged with wider spacing and the volume of deteriorated coal slightly grows as well. Stacking stockpiles in very proximity may reduce risk of self-heating hazard but would result a high concentration of carbonic gases zone at areas between two stockpiles, refer Fig. 20. If the three stockpiles were closely stacked in $5 \mathrm{~m}$ spacing, very high concentration $\mathrm{CO}_{2}$ would be present at these zones. Therefore more assessments should be implemented upon final decision of how wide the stockpiles should be located at a given context.

\subsubsection{Porosity or compaction}

Three porosity (i.e. $0.1,0.2,0.3$ ) are selected to represent three compaction extents of coal stockpile, namely densely packed, slightly packed, and loosely packed. Fig. 21 illustrates the maximum temperature rising profiles of the three stockpiles with the three compaction degrees. It is found the temperature rate significantly drops if stockpiles are densely packed due to high resistance to oxygen ingress. It spends 85 days to reach the critical temperature for stockpile $\mathrm{A}$ and it only reaches $340 \mathrm{~K}$ for stockpile B. Whilst for porosity 0.2 , it spends only 48 days for stockpile A to reach $363 \mathrm{~K}$ and only 
than that of loosely packed stockpile at the initial stage of coal oxidation. However after roughly one month the highest temperature of slightly packed stockpile surpasses that of loosely packed stockpile. A possible explanation is available oxygen of loose stockpile is more abundant than a slightly packed stockpile initially but with consumption and depletion of oxygen, it appears convective heat dissipation becomes dominant. This can be possibly demonstrated by Fig. 22 in which hot spot is moving deeper in more porous stockpile because of more heat being dissipated by convection while in deep stockpile oxygen concentration becomes too low to sustain a high rate of oxidation reaction. The abnormal effect of high porosity has been reported previously but at a slightly higher temperature [27]. In addition it can be seen the volume of oxidised coal substantially increases with more loosely packed stockpile. Therefore porosity less than 0.2 is recommended if coal is expected to be stored for a long run and porosity less than 0.3 is recommended if caking property and calorific value of coal needs to be retained. Densely packed stockpile has additional benefit due to its low emission of greenhouse effect gas, refer Fig. 23. The accumulation of $\mathrm{CO}_{2}$ is much alleviated between stockpile $\mathrm{A}$ and $\mathrm{B}$, and slightly diluted between stockpile B and C.

372 In Australia Hunter Valley power station pile height lower than $4.5 \mathrm{~m}$ is suggested for long run storage. To maintain the discreetness of the study, higher stockpiles $(5 \mathrm{~m}, 7.5 \mathrm{~m}, 10 \mathrm{~m})$ will be studied because it has been indicated higher stockpile is less safe in terms of coal spontaneous combustion [9, 13, 27]. As can be seen from Fig. 24, maximum temperature rising rate of stockpile A slightly slows down with greater height but the effects are limited. Maximum temperature rising rate of stockpile B and C also slightly slow down with lower height for a long run but initially lower stockpile may have faster temperature rising rate. For example, maximum temperature of $10 \mathrm{~m}$ high stockpile B falls behind both $5 \mathrm{~m}$ and $7.5 \mathrm{~m}$ high stockpile B in the first 20 days. A possible reason is lower stockpile A weakens the "protection" to stockpile B and more air is attracted into stockpile B. With progress of self-heating, it becomes increasingly hard for lower stockpile B to contain the interior thermal energy and cause slow temperature rising rate after an initial period. From Fig. 25 it can be known the effects of different heights of stockpile on temperature contour and volume of deteriorated coal are very limited at low temperature range. The dispersion of $\mathrm{CO}_{2}$ in farfield with various stockpile heights is also studied. As can be seen from Fig. $26 \mathrm{CO}_{2}$ is more readily to be diluted for lower stockpile because more airflow is introduced towards stockpile B and C due to less obstruction of stockpile A. Through above analysis it can be deducted lower stockpile can have two benefits: (i) reduce self-heating risk; (ii) facilitate dispersion of 
Three slopes are studied in this work, namely $35^{\circ}, 50^{\circ}$ (the base model), and $65^{\circ}$ representing gentle, normal, and steep stockpile respectively. It has been reported the self-heating problem can be alleviated when the slope is made gentler [27, 30]. This study indicates a similar finding but merely for the first stockpile at low temperature range. As can be seen from Fig. 27, side slope has a strong effect on maximum temperature rising rate of stockpile A but has limited influence on stockpile B and C. Self-heating rate of stockpile A significantly reduces with gentler side slope. When stockpile is $35^{\circ}$, it is found initial self-heating rate of gentle stockpile B is faster than its steep stockpile but slows down for a long run. A possible reason is "protection" of stockpile A is considerably weakened with gentle slope and as a result of it, more air is directed to stockpile B. Fig. 28shows the evolution of hot spot with different slopes at 30 day. It can be seen hot zone of stockpile A is enlarged with steeper slope and little difference can be observed for stockpile B and C. In addition it appears volume of coal being oxidised slightly increases with steep side slope for stockpile A and little difference can be identified for stockpile $\mathrm{B}$ and $\mathrm{C}$. The dispersion pattern of $\mathrm{CO}_{2}$ with different stockpile slopes is also investigated. As can be seen from Fig. 29, the high concentration of $\mathrm{CO}_{2}$ gas mixture can be better flushed with steep slope stockpile and therefore it needs to be further evaluated upon employing a gentle slope to alleviate the self-heating problem.

\section{Conclusion}

The phenomenon of self-heating of coal stockpile is a result of complex physical and chemical processes. Although this is a long-standing problem, complete understanding has never been fully grasped. To gain more insights of self-heating hazard on coal stockpile especially for the scenario where multiple stockpiles need to be constructed in proximity, a nonequilibrium thermal model is developed with assistance of CFD code under conditions of wind flow. After validation of the proposed model a base model is selected and investigated in many details. It was found wind velocity builds up as it encounters the first stockpile and then significantly drops at the regions between two stockpiles where a wake region is expected to be induced. Exterior pressure drop around the three stockpiles is studied to provide more understanding of fluid dynamics involved in this problem. Interior pressure gradients distribution for the three stockpiles is further investigated to estimate the possible travel path of airflow in a more fundamental way. All the evidence indicates more airflow will be introduced into stockpile A than the other two stockpiles and therefore it is postulated stockpile A is like a wind barrier and very likely to lead the evolution of self-heating. The conjecture was then confirmed by maximum temperature rising profiles of the three stockpiles. The progressive consumption of oxygen and accumulation of gaseous products are also analysed to gain more insights in coal oxidation process. Liberation of carbonic gas and its flow pattern in the farfield is investigated to grasp more knowledge as greenhouse effect gas emission contributed from low temperature and spontaneous combustion of coal stockpile has attracted intensive attentions recently. Last not the least parametric analysis was conducted and several meaningful findings are listed: 
a) Stronger wind would promote evolution of self-heating hazard and increase volume of deteriorated coal but meanwhile facilitate dilution of the gaseous products;

b) Different spacing has little influence on self-heating behaviour of the first stockpile and more closely stacked 


\section{Reference list}

1. Wang, H., B.Z. Dlugogorski, and E.M. Kennedy, Coal oxidation at low temperatures: oxygen consumption, oxidation products, reaction mechanism and kinetic modelling. Progress in Energy and Combustion Science, 2003. 29(6): p. 487-513.

2. Kaji, R., Y. Hishinuma, and Y. Nakamura, Low temperature oxidation of coals-a calorimetric study. Fuel, 1987. 66(2): p. 154-157.

3. Itay, M., C.R. Hill, and D. Glasser, A study of the low temperature oxidation of coal. Fuel Processing Technology, 1989. 21(2): p. 81-97.

4. Shamsi, A., L.J. Shadle, and K.S. Seshadri, Study of low-temperature oxidation of buckskin subbituminous coal and derived chars produced in ENCOAL process. Fuel Processing Technology, 2004. 86(3): p. 275-292.

5. Yuan, L. and A.C. Smith, The effect of ventilation on spontaneous heating of coal. Journal of Loss Prevention in the Process Industries, 2012. 25(1): p. 131-137.

6. Beamish, B.B., M.A. Barakat, and J.D.S. George, Adiabatic testing procedures for determining the self-heating propensity of coal and sample ageing effects. Thermochimica Acta, 2000. 362(1-2): p. 79-87.

7. Beamish, B.B., M.A. Barakat, and J.D. St. George, Spontaneous-combustion propensity of New Zealand coals under adiabatic conditions. International Journal of Coal Geology, 2001. 45(2-3): p. 217-224.

8. Beamish, B.B. and D.G. Blazak, Relationship between ash content and R70 self-heating rate of Callide Coal. International Journal of Coal Geology, 2005. 64(1-2): p. 126-132.

9. Zhu, H.-q., Z.-y. Song, B. Tan, and Y.-z. Hao, Numerical investigation and theoretical prediction of selfignition characteristics of coarse coal stockpiles. Journal of Loss Prevention in the Process Industries, 2013. 26(1): p. 236-244.

10. Arisoy, A. and B. Beamish, Mutual effects of pyrite and moisture on coal self-heating rates and reaction rate data for pyrite oxidation. Fuel, 2015. 139: p. 107-114.

11. Arisoy, A. and B. Beamish, Reaction kinetics of coal oxidation at low temperatures. Fuel, 2015. 159: p. $412-417$.

12. Carras, J.N. and B.C. Young, Self-heating of coal and related materials: Models, application and test methods. Progress in Energy and Combustion Science, 1994. 20(1): p. 1-15.

13. Arisoy, A. and F. Akgün, Effect of Pile Height on Spontaneous Heating of Coal Stockpiles. Combustion Science and Technology, 2000. 153(1): p. 157-168.

14. Yang, Y., Z. Li, Y. Tang, Z. Liu, and H. Ji, Fine coal covering for preventing spontaneous combustion of coal pile. Natural Hazards, 2014. 74(2): p. 603-622.

15. Kuenzer, C., J. Zhang, A. Tetzlaff, P. van Dijk, S. Voigt, H. Mehl, and W. Wagner, Uncontrolled coal fires and their environmental impacts: Investigating two arid mining regions in north-central China. Applied Geography, 2007. 27(1): p. 42-62.

16. Carras, J.N., S.J. Day, A. Saghafi, and D.J. Williams, Greenhouse gas emissions from low-temperature oxidation and spontaneous combustion at open-cut coal mines in Australia. International Journal of Coal Geology, 2009. 78(2): p. 161-168.

17. Day, S., J. Carras, R. Fry, and D. Williams, Greenhouse gas emissions from Australian open-cut coal mines: contribution from spontaneous combustion and low-temperature oxidation. Environmental Monitoring \& Assessment, 2010. 166(1-4): p. 529-541.

18. Chen, G., X. Ma, M. Lin, X. Peng, and Z. Yu, Pollutant emission characteristics and interaction during lowtemperature oxidation of blended coal. Journal of the Energy Institute, 2016. 89(1): p. 40-47.

19. Sondreal, E.A. and R.C. Ellman, Laboratory determination of factors affecting storage of North Dakota lignite: computer simulation of spontaneous heating. 1974, Bureau of Mines, Grand Forks, N. Dak.(USA). Grand Forks Energy Research Lab.

20. Nordon, P., A model for the self-heating reaction of coal and char. Fuel, 1979. 58(6): p. $456-464$.

21. Schmal, D., J.H. Duyzer, and J.W. van Heuven, A model for the spontaneous heating of coal. Fuel, 1985. 64(7): p. 963-972.

22. Brooks, K., S. Bradshaw, and D. Glasser, Spontaneous combustion of coal stockpiles - an unusual chemical reaction engineering problem. Chemical Engineering Science, 1988. 43(8): p. 2139-2145.

23. Brooks, K. and D. Glasser, A simplified model of spontaneous combustion in coal stockpiles. Fuel, 1986. 65(8): p. 1035-1041.

24. Young, B.D., D.F. Williams, and A.W. Bryson, Two-dimensional natural convection and conduction in a packed bed containing a hot spot and its relevance to the transport of air in a coal dump. International Journal of Heat and Mass Transfer, 1986. 29(2): p. 331-336.

25. Bradshaw, S., D. Glasser, and K. Brooks, Self-ignition and convection patterns in an infinite coal layer. Chemical Engineering Communications, 1991. 105(1): p. 255-278.

26. Arisoy, A. and F. Akgün, Modelling of spontaneous combustion of coal with moisture content included. Fuel, 1994. 73(2): p. 281-286.

27. Akgun, F. and R.H. Essenhigh, Self-ignition characteristics of coal stockpiles: theoretical prediction from a two-dimensional unsteady-state model. Fuel, 2001. 80(3): p. 409-415. 
2

28. Edwards, J.C., Mathematical modeling of spontaneous heating of a coalbed. Report of investigations (United States. Bureau of Mines) ; 9296., ed. M. United States. Bureau of. 1990, Washington, D.C. : U.S. Dept. of the Interior, Bureau of Mines.

29. Salinger, A.G., R. Aris, and J.J. Derby, Modeling the spontaneous ignition of coal stockpiles. AIChE Journal, 1994. 40(6): p. 991-1004.

30. Krishnaswamy, S., P.K. Agarwal, and R.D. Gunn, Low-temperature oxidation of coal. 3. Modelling spontaneous combustion in coal stockpiles. Fuel, 1996. 75(3): p. 353-362.

31. Hull, A., J.L. Lanthier, and P.K. Agarwal, The role of the diffusion of oxygen in the ignition of a coal stockpile in confined storage. Fuel, 1997. 76(10): p. 975-983.

32. Hull, A.S., J.L. Lanthier, Z. Chen, and P.K. Agarwal, The role of the diffusion of oxygen and radiation on the spontaneous combustibility of a coal pile in confined storage. Combustion and Flame, 1997. 110(4): p. 479-493.

33. Monazam, E.R., L.J. Shadle, and A. Shamsi, Spontaneous combustion of char stockpiles. Energy \& fuels, 1998. 12(6): p. 1305-1312.

34. Moghtaderi, B., B.Z. Dlugogorski, and E.M. Kennedy, Effects of Wind Flow on Self-Heating Characteristics of Coal Stockpiles. Process Safety and Environmental Protection, 2000. 78(6): p. 445-453.

35. Yuan, L. and A.C. Smith, Numerical study on effects of coal properties on spontaneous heating in longwall gob areas. Fuel, 2008. 87(15-16): p. 3409-3419.

36. Yuan, L. and A.C. Smith, CFD modeling of spontaneous heating in a large-scale coal chamber. Journal of Loss Prevention in the Process Industries, 2009. 22(4): p. 426-433.

37. Ejlali, A., D.J. Mee, K. Hooman, and B.B. Beamish, Numerical modelling of the self-heating process of a wet porous medium. International Journal of Heat and Mass Transfer, 2011. 54(25-26): p. 5200-5206.

38. Taraba, B. and Z. Michalec, Effect of longwall face advance rate on spontaneous heating process in the gob area-CFD modelling. Fuel, 2011. 90(8): p. 2790-2797.

39. Taraba, B., Z. Michalec, V. Michalcová, T. Blejchař, M. Bojko, and M. Kozubková, CFD simulations of the effect of wind on the spontaneous heating of coal stockpiles. Fuel, 2014. 118: p. 107-112.

40. Kim, C.J. and C.H. Sohn, A novel method to suppress spontaneous ignition of coal stockpiles in a coal storage yard. Fuel Processing Technology, 2012. 100: p. 73-83.

41. Xia, T., X. Wang, F. Zhou, J. Kang, J. Liu, and F. Gao, Evolution of coal self-heating processes in longwall gob areas. International Journal of Heat and Mass Transfer, 2015. 86: p. 861-868.

42. Shi, T., X. Wang, J. Deng, and Z. Wen, The mechanism at the initial stage of the room-temperature oxidation of coal. Combustion and Flame, 2005. 140(4): p. 332-345.

43. Nordon, P. and N.W. Bainbridge, Heat of wetting of a bituminous coal. Fuel, 1983. 62(5): p. 619-621.

44. Clemens, A.H. and T.W. Matheson, The role of moisture in the self-heating of low-rank coals. Fuel, 1996. 75(7): p. 891-895.

45. McCutcheon, A.L. and M.A. Wilson, Low-Temperature Oxidation of Bituminous Coal and the Influence of Moisture. Energy \& fuels, 2003. 17(4): p. 929-933.

46. Chen, X.D. and J.B. Stott, The effect of moisture content on the oxidation rate of coal during near-equilibrium drying and wetting at $50{ }^{\circ} \mathrm{C}$. Fuel, 1993. 72(6): p. 787-792.

47. Bowes, P.C., Self-heating : evaluating and controlling the hazards. 1984, Dept. of the Environment, Building Research Establishment ;: Garston, Merseyside :.

48. Krajčiová, M., L. Jelemenský, M. Kiša, and J. Markoš, Model predictions on self-heating and prevention of stockpiled coals. Journal of Loss Prevention in the Process Industries, 2004. 17(3): p. 205-216.

49. Wang, G., T. Ren, K. Wang, and A. Zhou, Improved apparent permeability models of gas flow in coal with Klinkenberg effect. Fuel, 2014. 128: p. 53-61.

50. $\quad$ ANSYS FLUENT 15.0, Users' guide, ANSYS Inc. 2014.

51. Bird, R.B., W.E. Stewart, and E.N. Lightfoot, Transport phenomena, John Wiley and Sons, Inc., New York (1960). .

52. McCaughey, M. and D.F. Fletcher, Calculations of the wind-induced pressure distribution on a model building. Fire Safety Journal, 1993. 21(3): p. 189-205.

53. Fierro, V., J.L. Miranda, C. Romero, J.M. Andrés, A. Arriaga, D. Schmal, and G.H. Visser, Prevention of spontaneous combustion in coal stockpiles: Experimental results in coal storage yard. Fuel Processing Technology, 1999. 59(1): p. 23-34. 


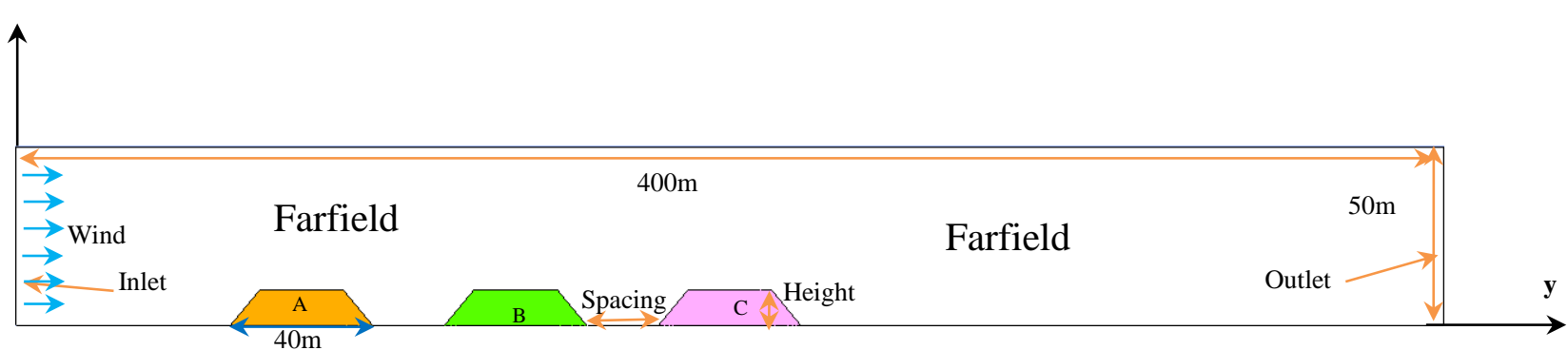

570

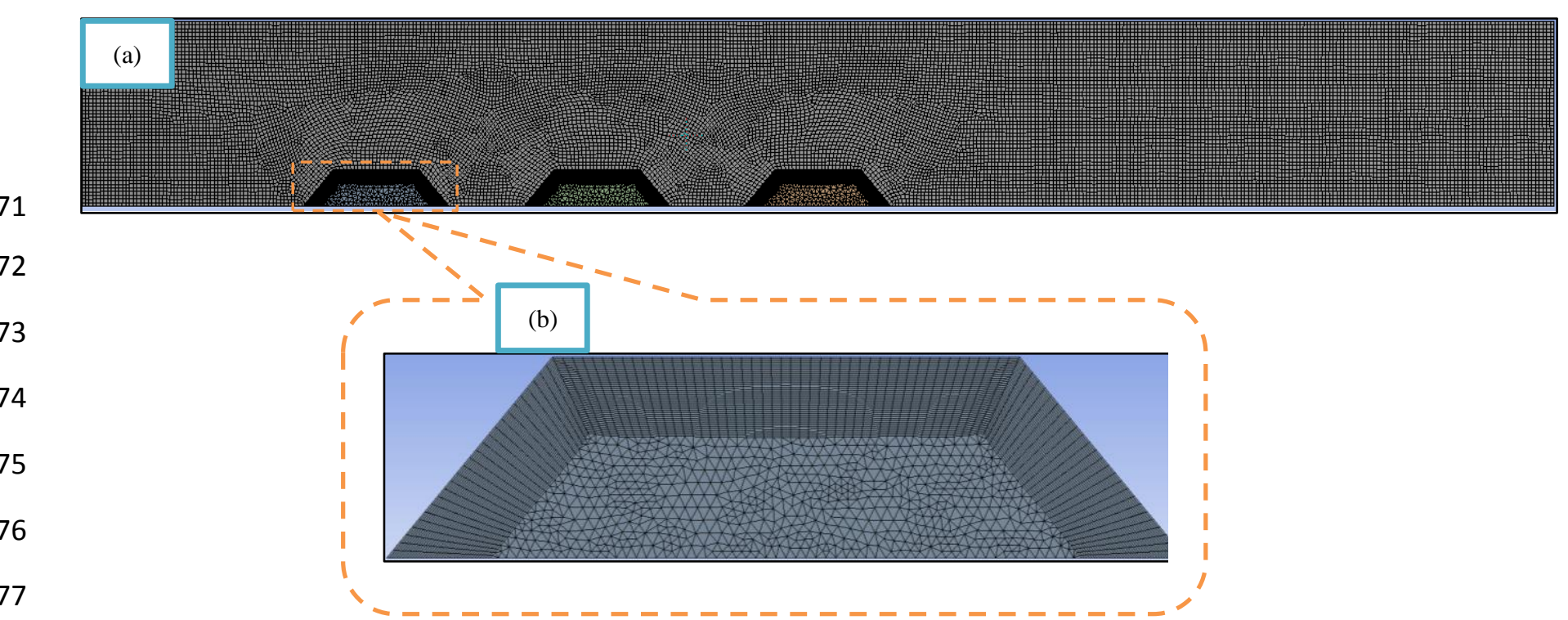

Fig. 1 A schematic overview of the simulation

Fig. 2 Mesh used in the simulation: (a) an overview; (b) a close-up view of stockpile A

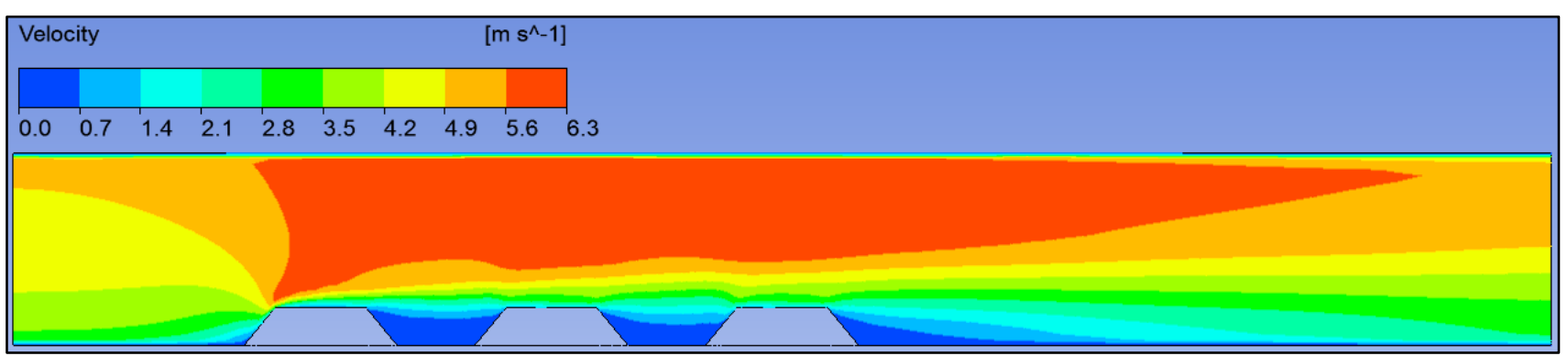




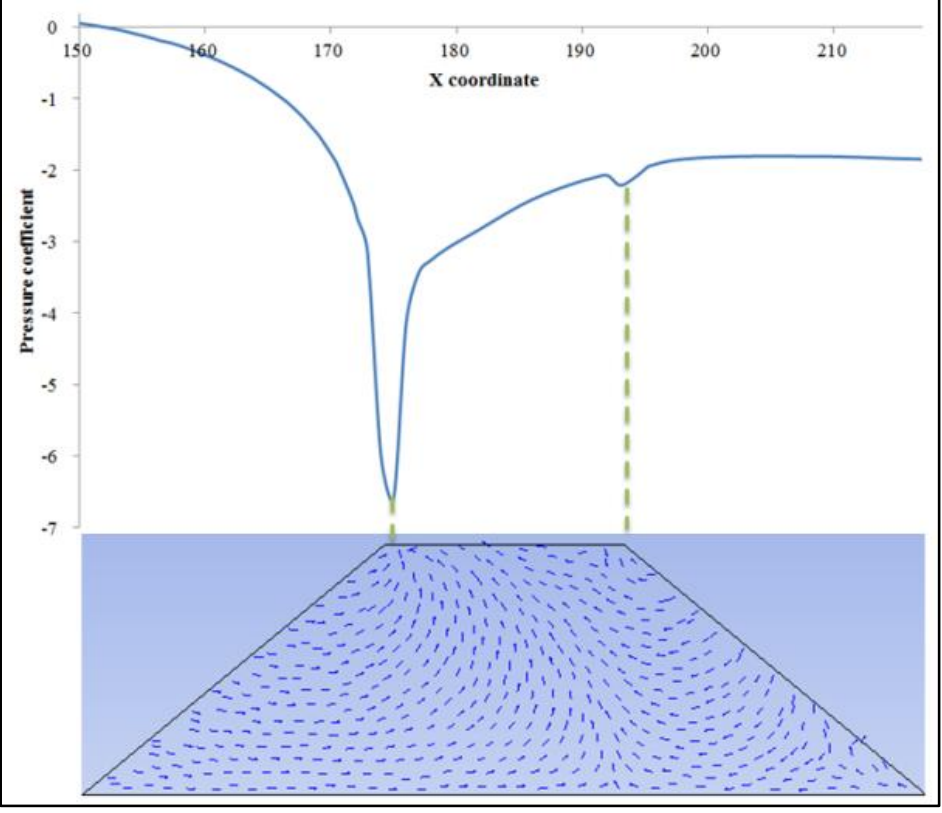

(a) Validation model

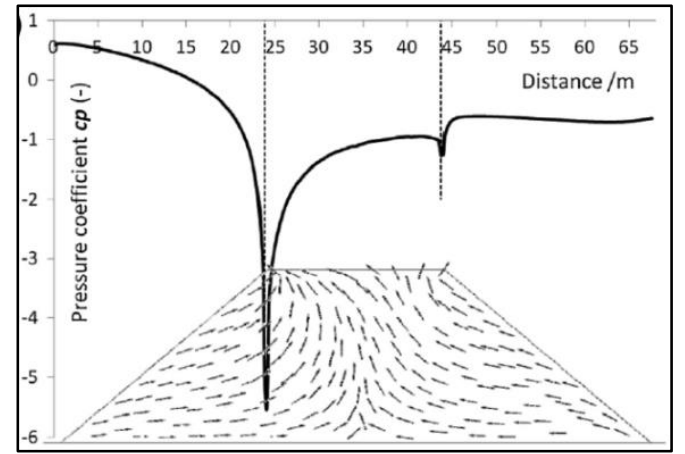

(b) Taraba's model [39]

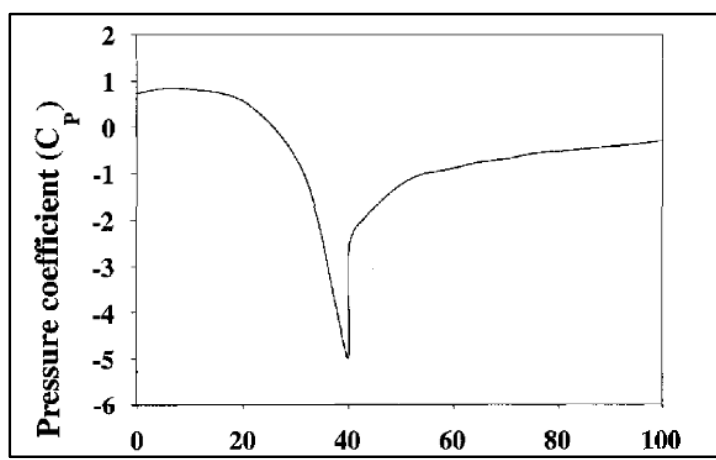

Fig. 4 The variation of the pressure coefficient around stockpile

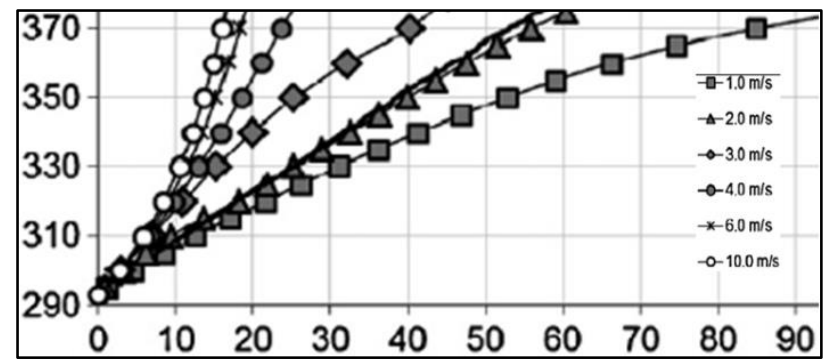

(a) Taraba's model [39]

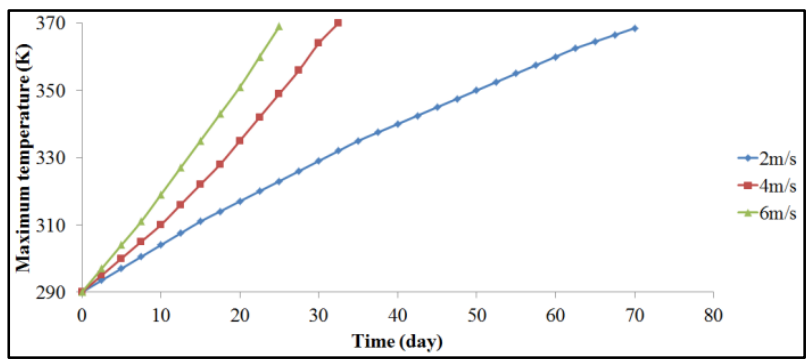

(b)Validation model

Fig. 5 Validation of maximum temperature rising profile 


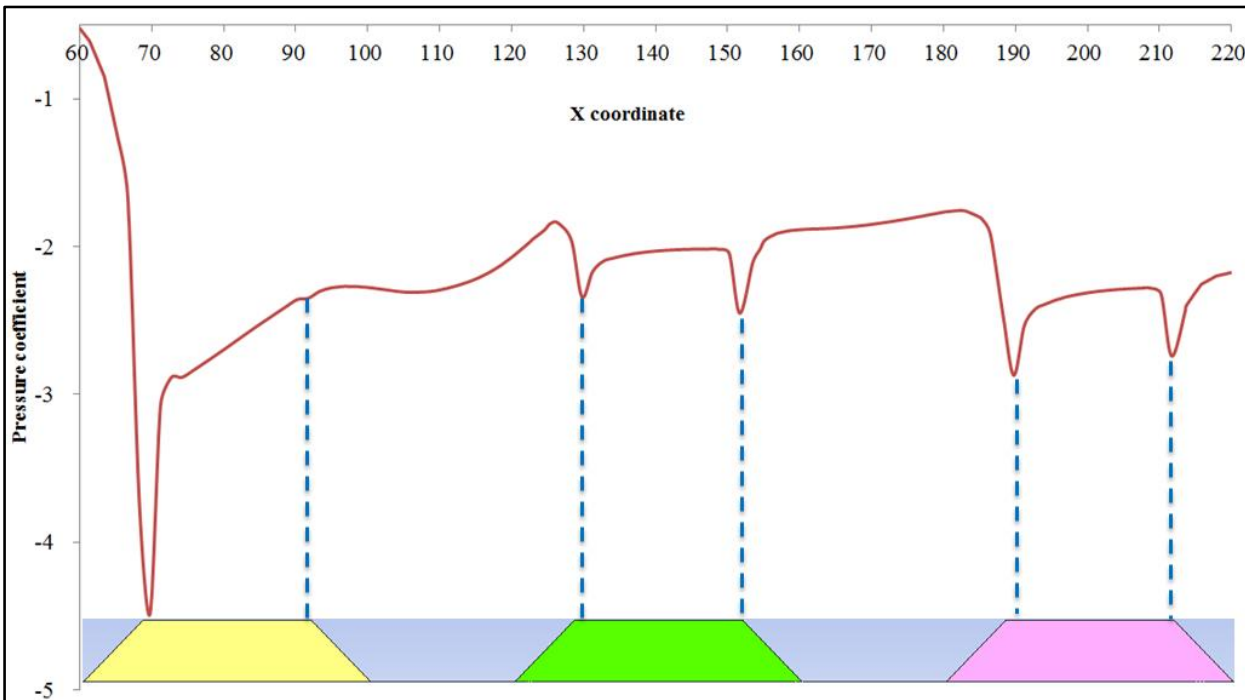

599

Fig. 7 Distribution of the pressure coefficient around the three stockpiles for the base model
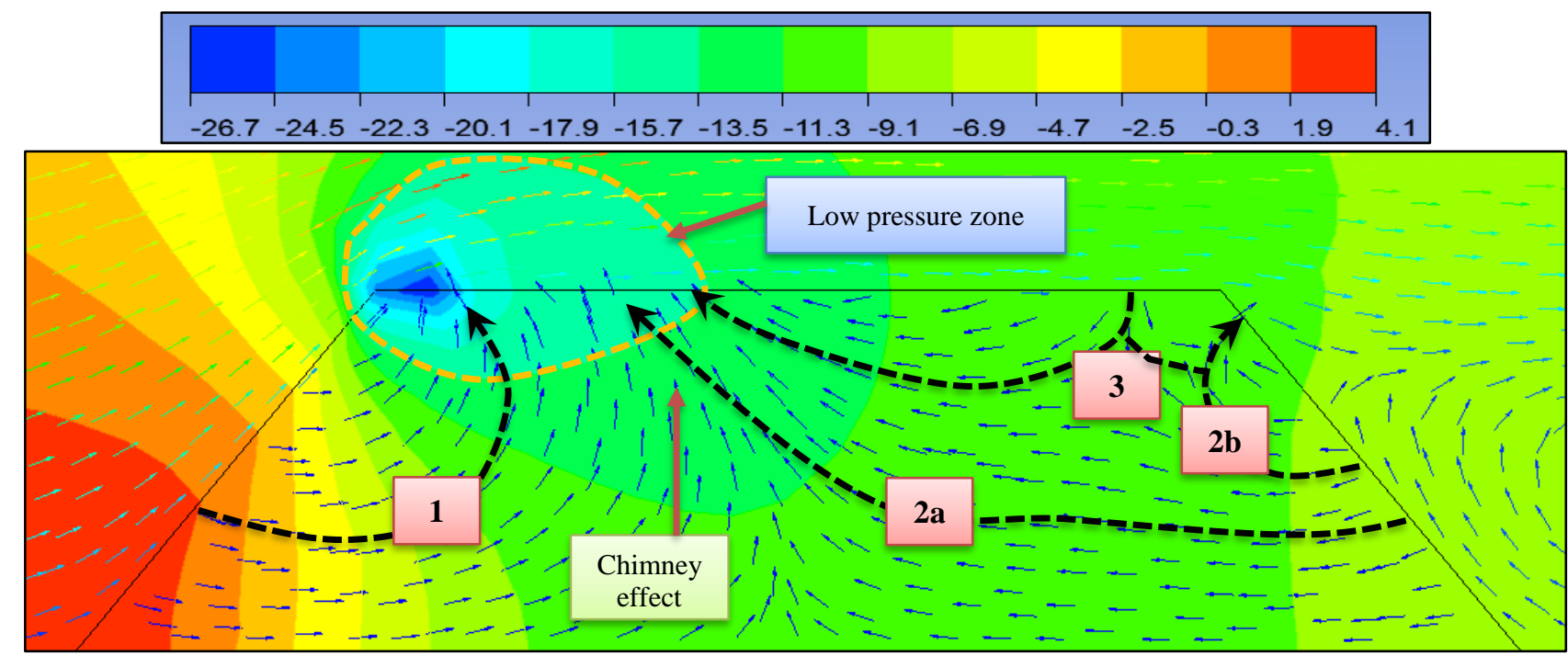

(a) Stockpile A

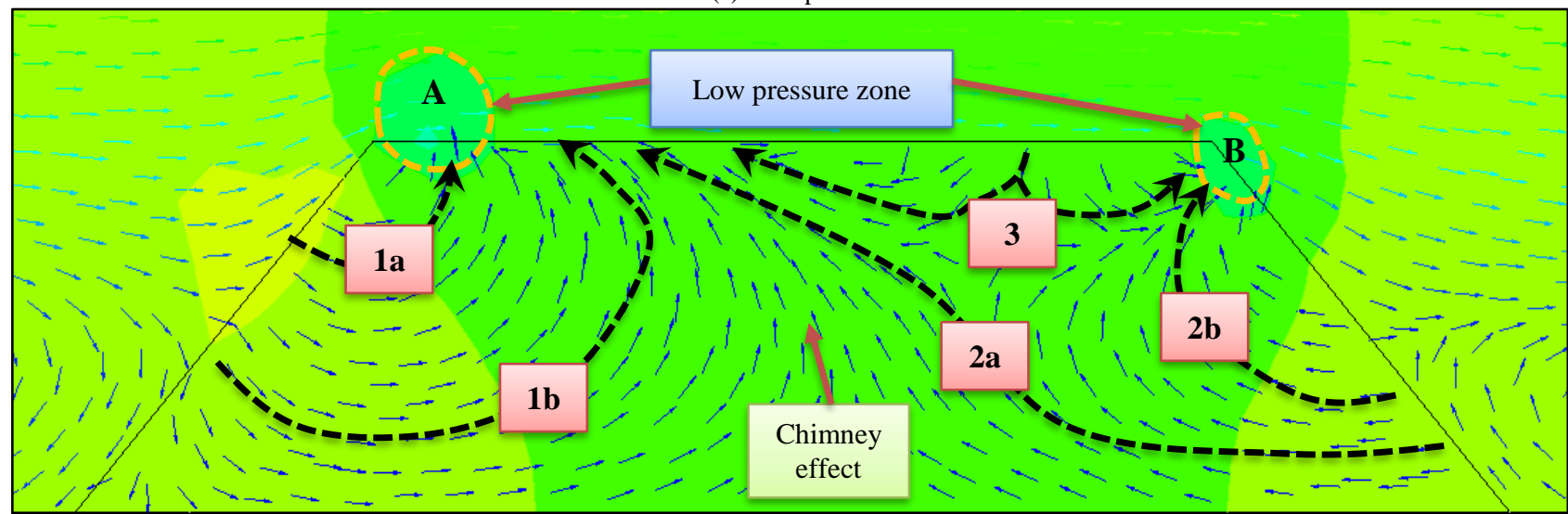

(b) Stockpile B 


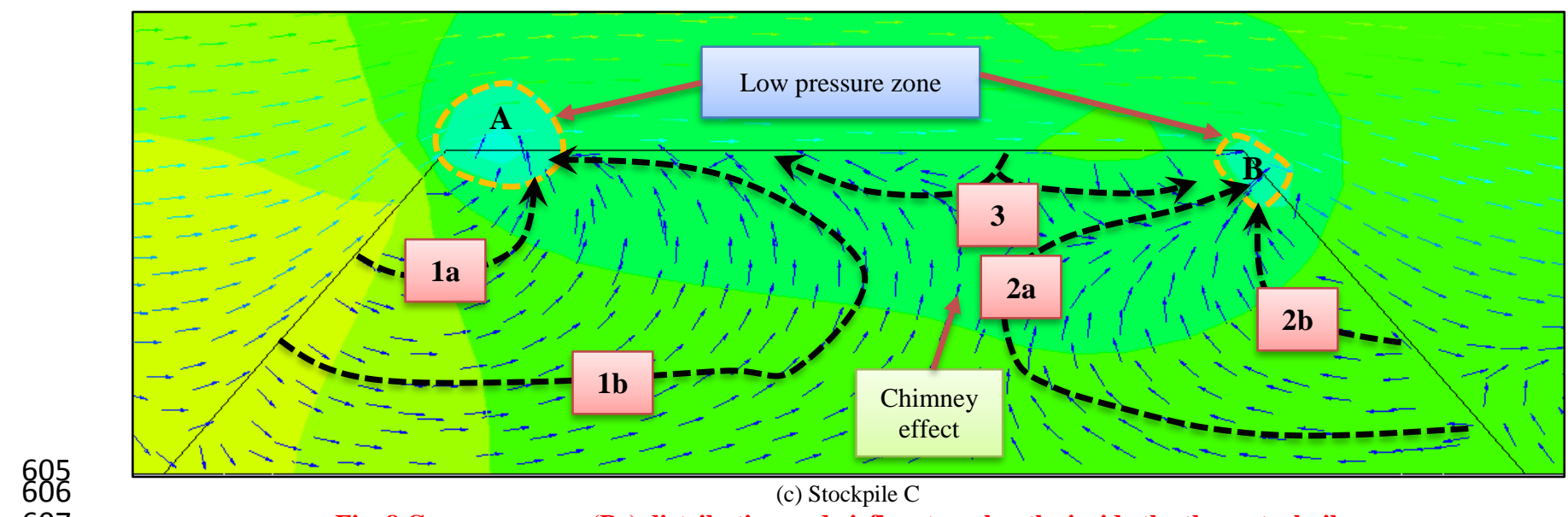

Fig. 8 Gauge pressure (Pa) distribution and airflow travel paths inside the three stockpiles
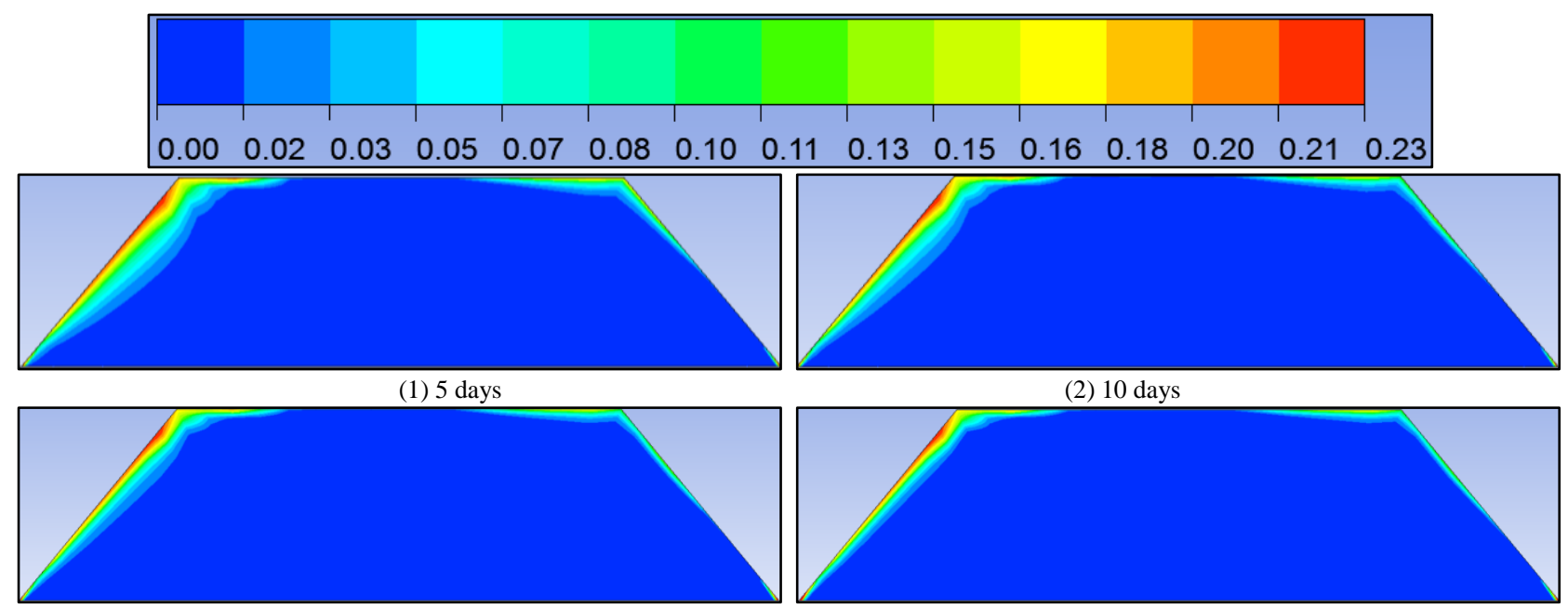

(3) 15 days

(a) Stockpile A
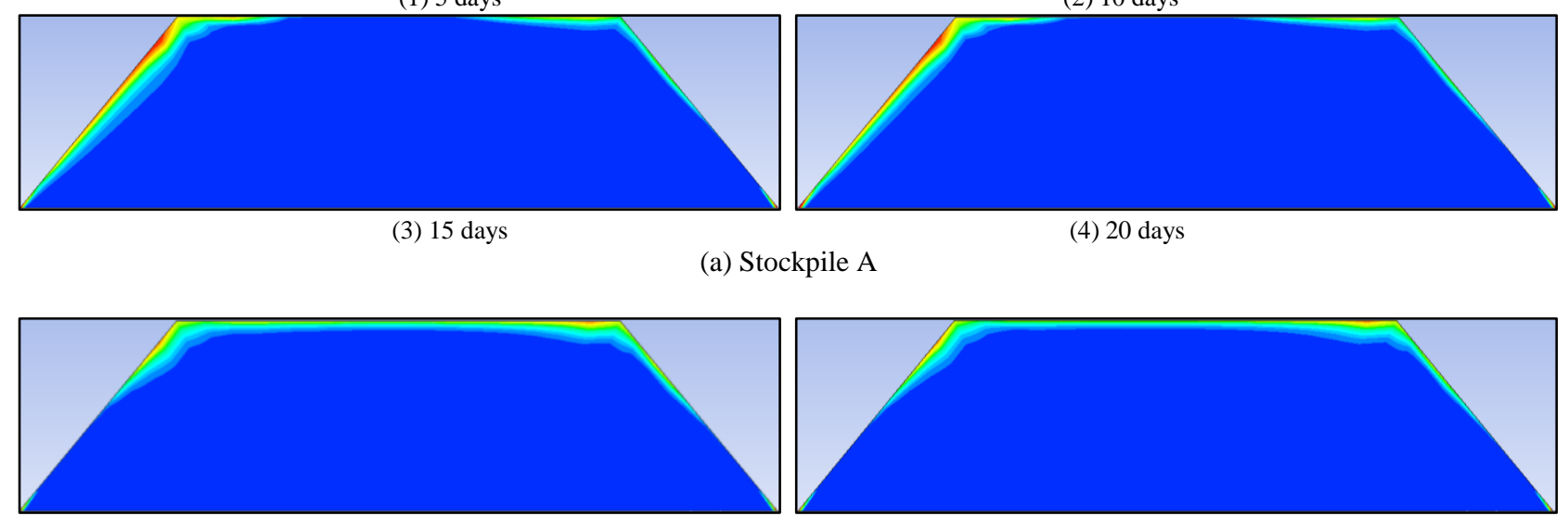

(1) 5 days
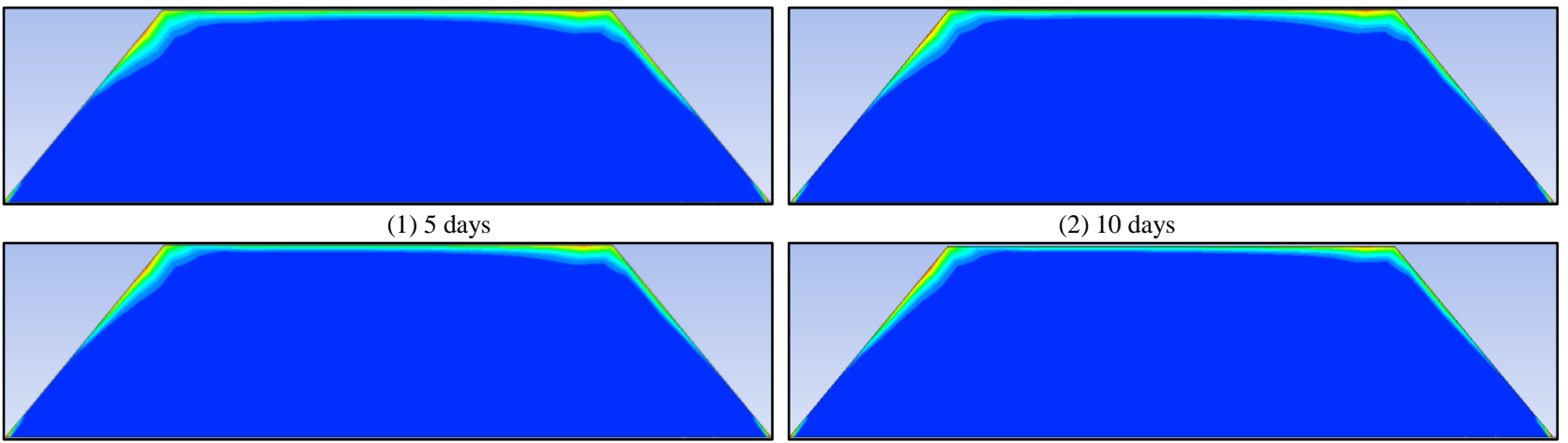

(2) 10 days

(b) Stockpile B

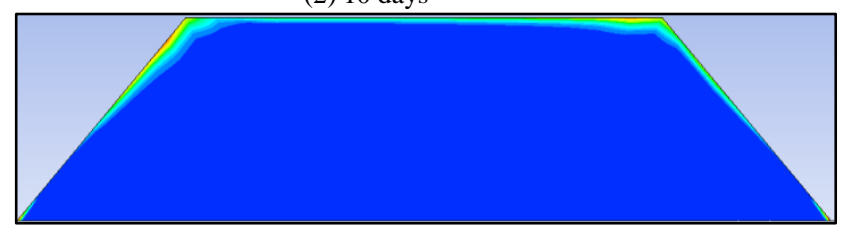

(4) 20 days

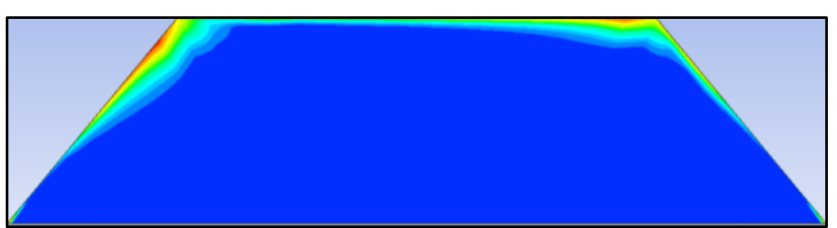

(1) 5 days

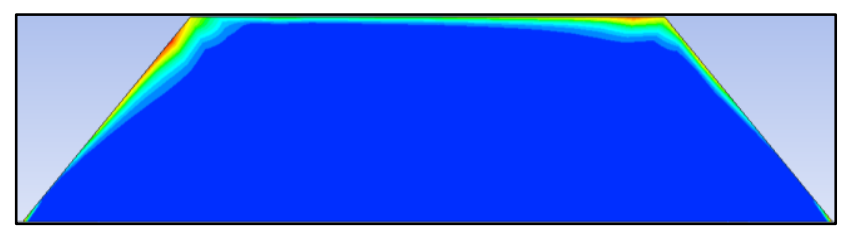

(2) 10 days 


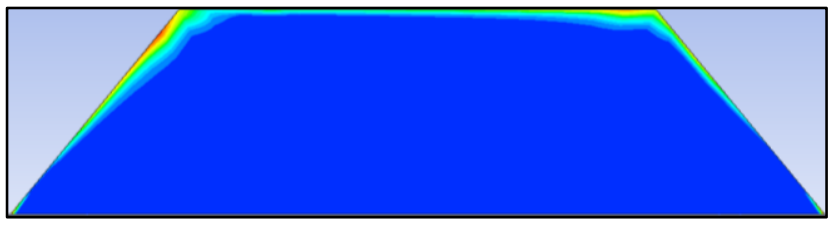

(3) 15 days

(c) Stockpile C

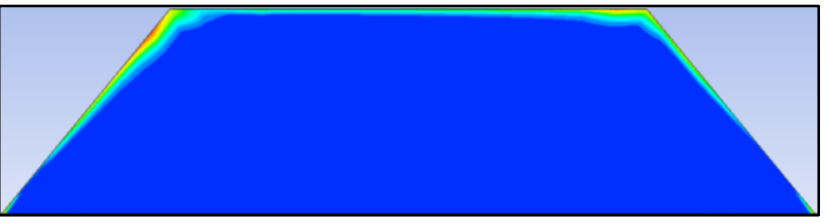

(4) 20 days

Fig. 9 Oxygen contours (mass fraction) of three stockpiles of the base model after various periods

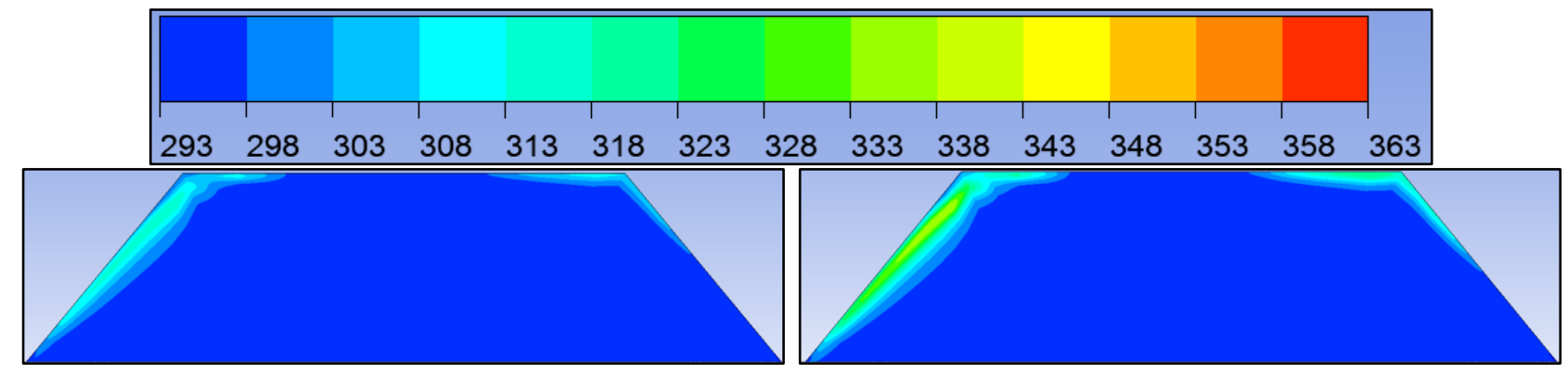

(1) 10 days

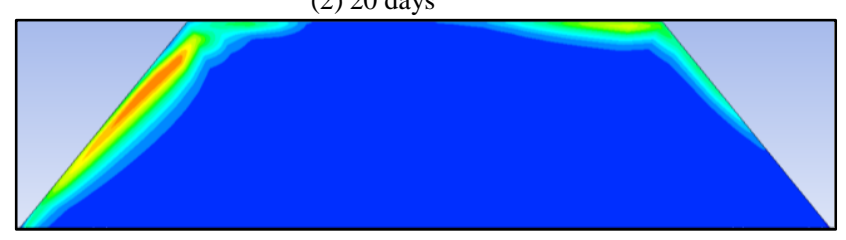

(3) 30 days

(4) 40 days

(a) Stockpile A

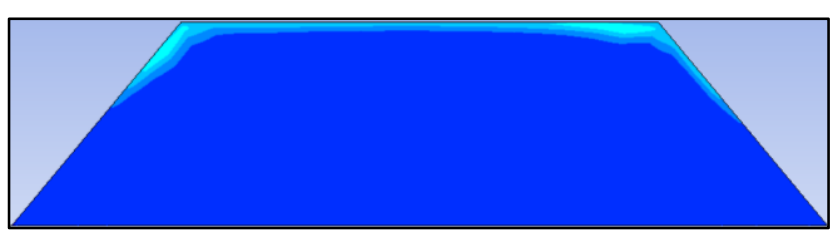

(1) 10 days
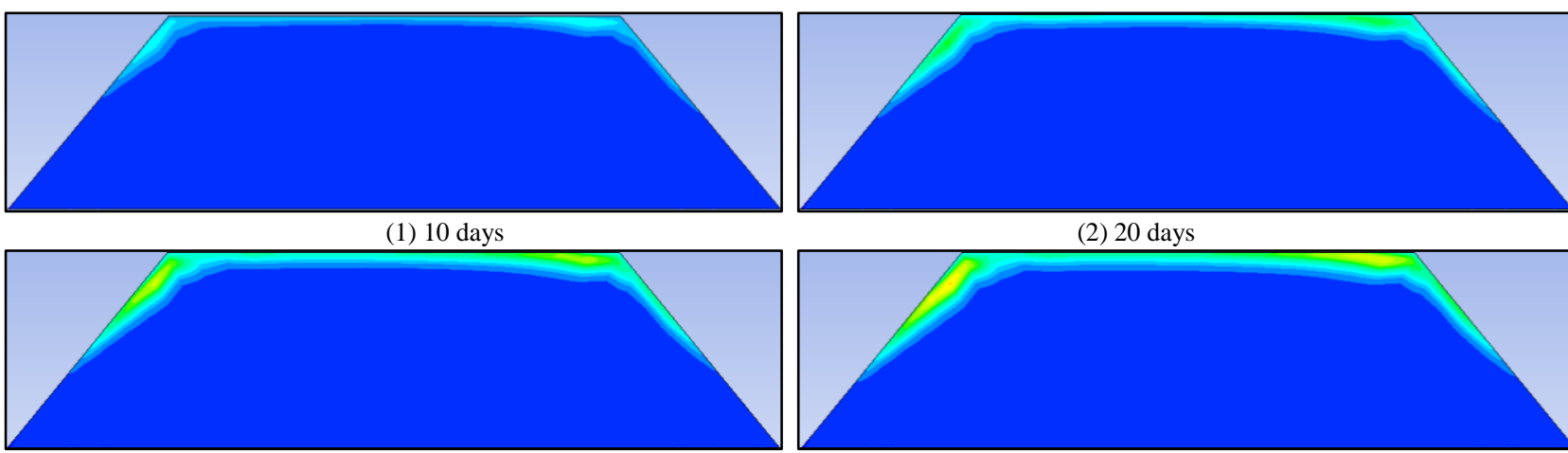

(3) 30 days

(b) Stockpile B

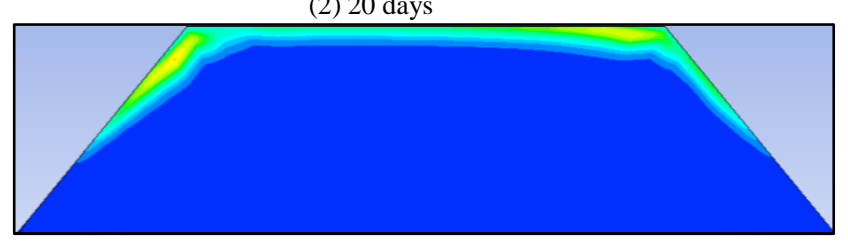

(4) 40 days

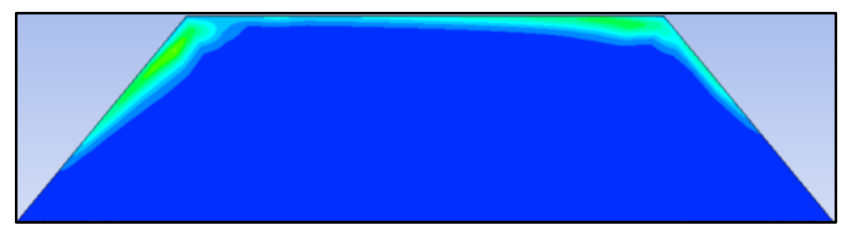

(1) 10 days

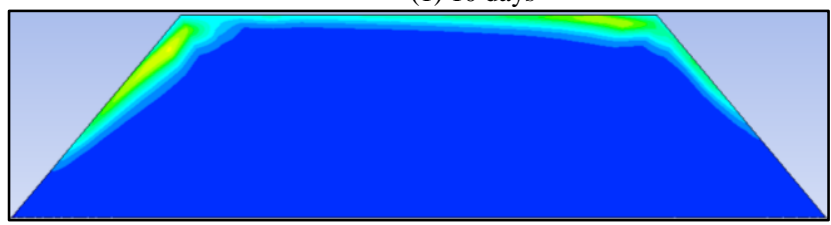

(3) 30 days

(c) Stockpile C

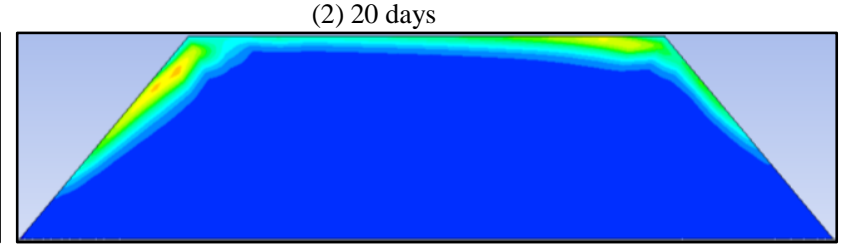

(4) 40 days

Fig. 10 Temperature (K) distribution of the three stockpiles of the base model after various periods 


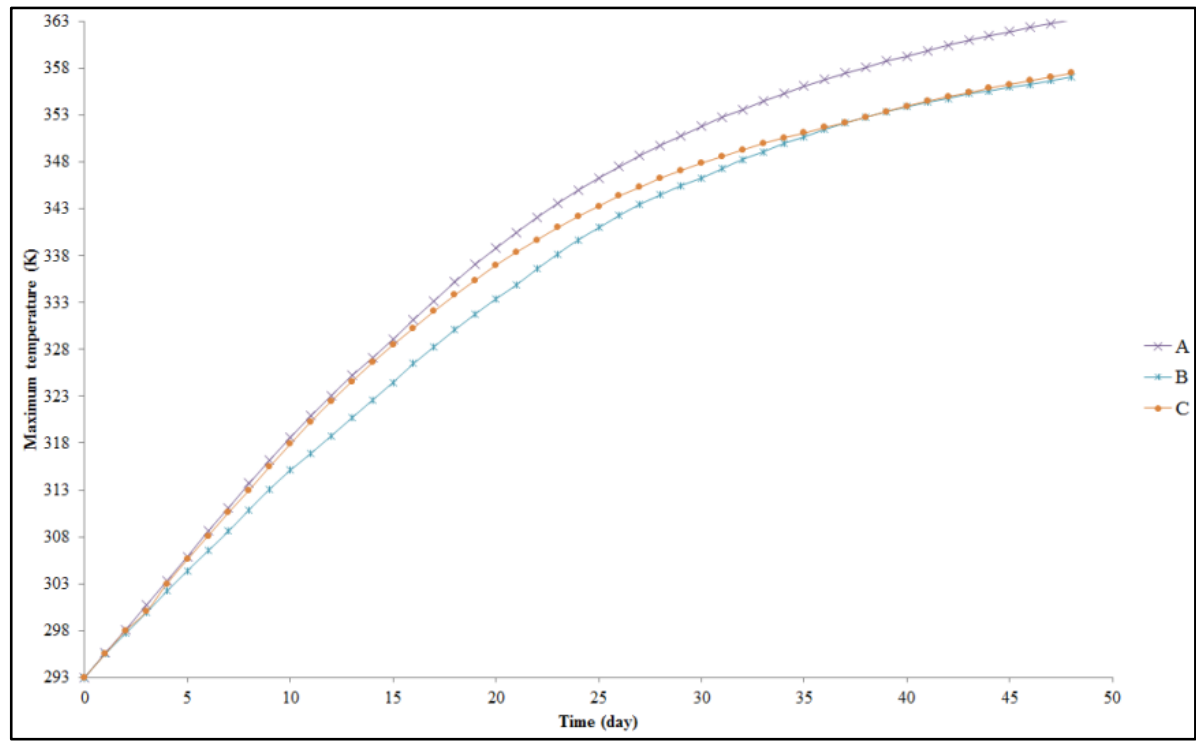

Fig. 11 The highest temperature rising (K) profiles of the three stockpiles of the base model
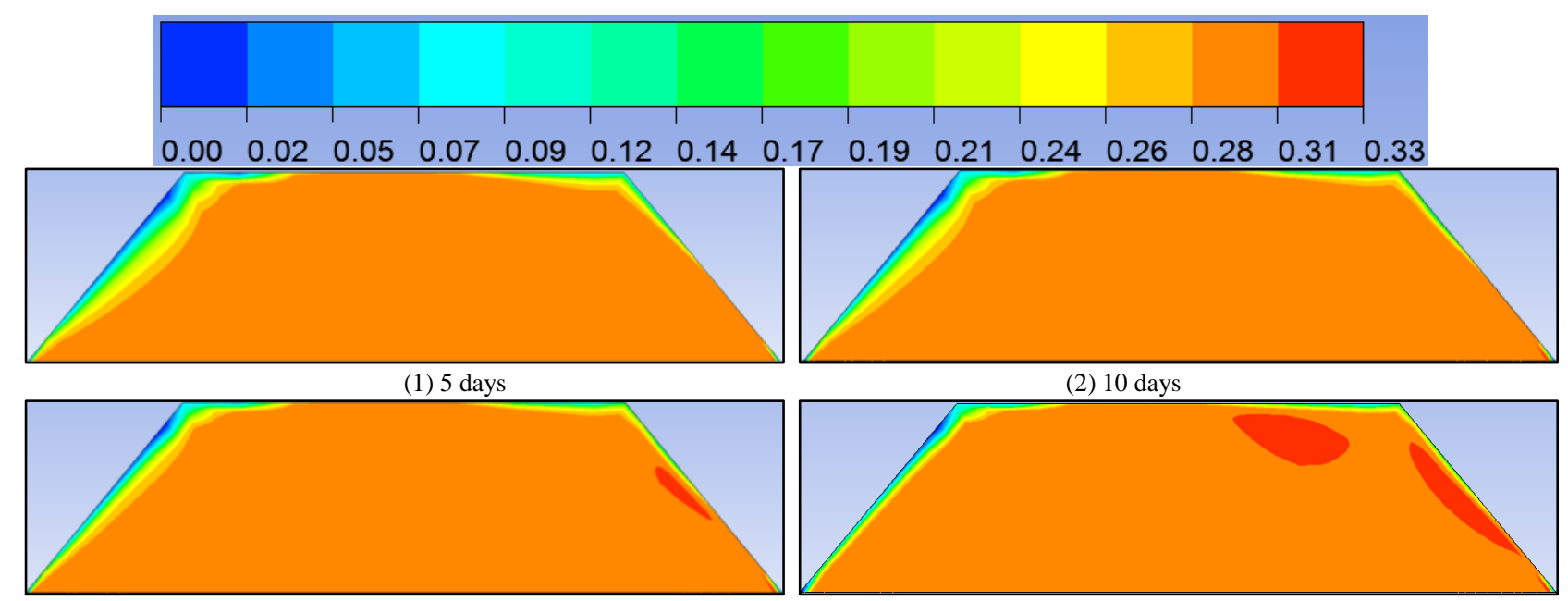

(3) 15 days

(a) Stockpile A

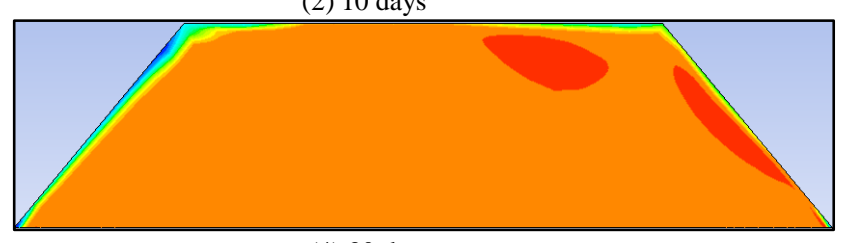

(4) 30 days

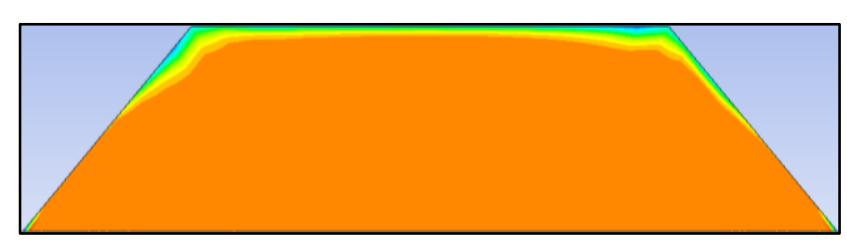

(1) 5 days
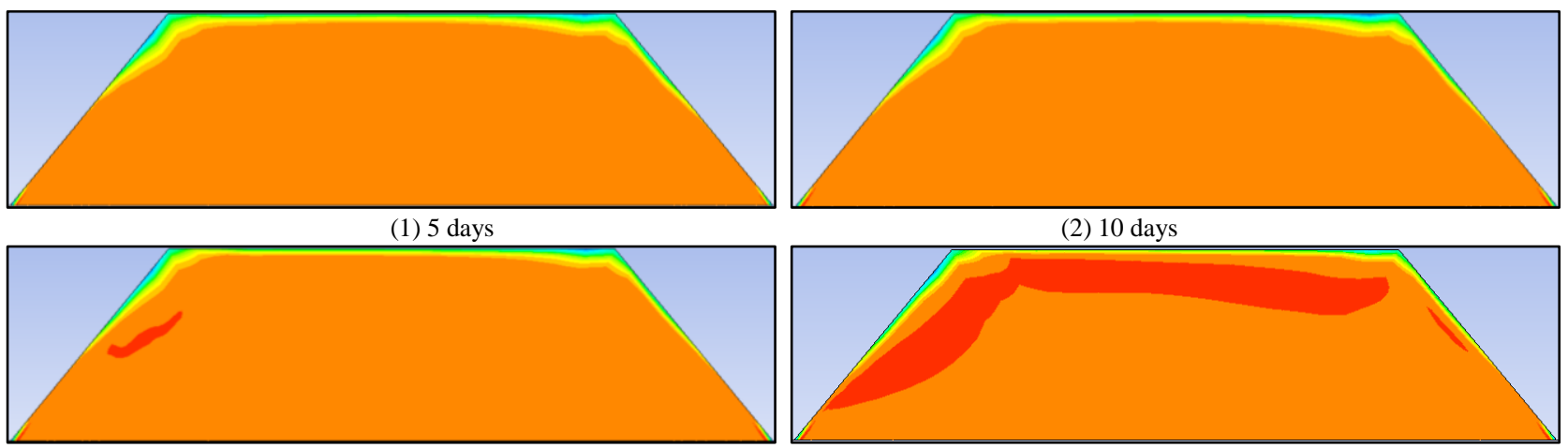

(3) 15 days

(b) Stockpile B

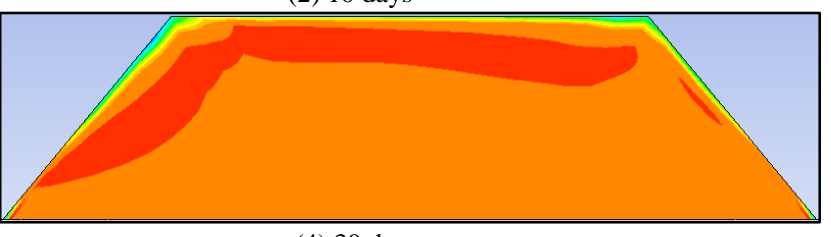

(4) 30 days
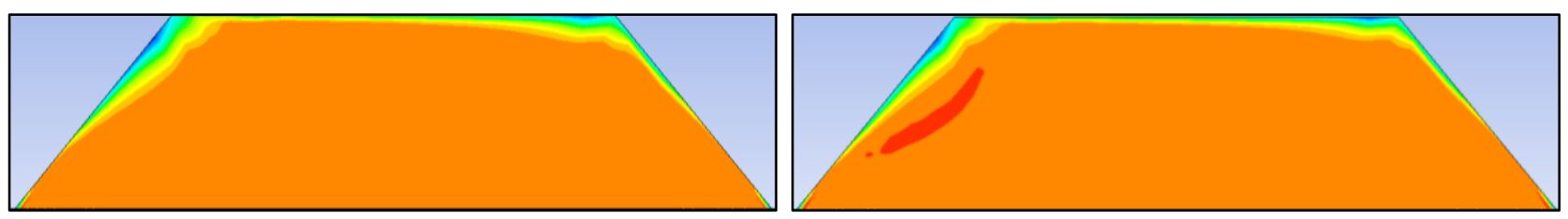
(1) 5 days

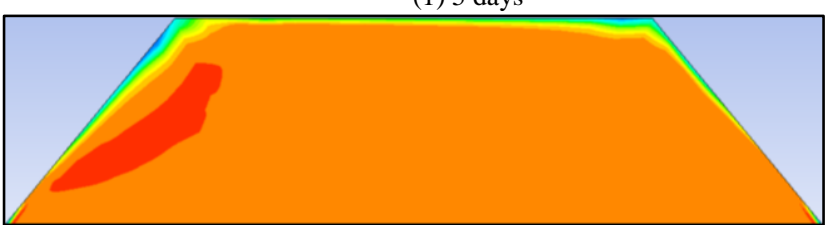

(3) 15 days

(c) Stockpile C

(2) 10 days

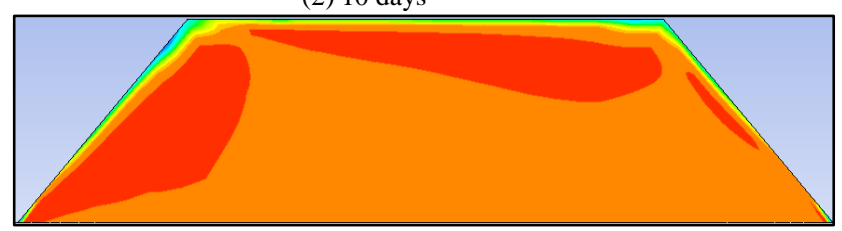

(4) 30 days

Fig. $12 \mathrm{CO}_{2}$ contours (mass fraction) of three stockpiles of the base model after various periods

671

672

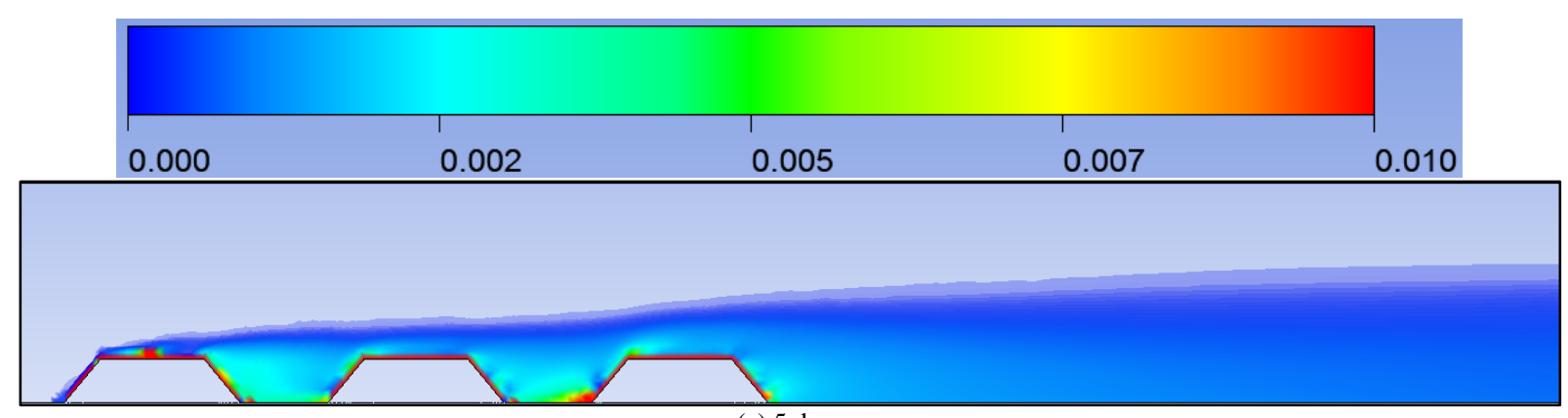

(a) 5 days

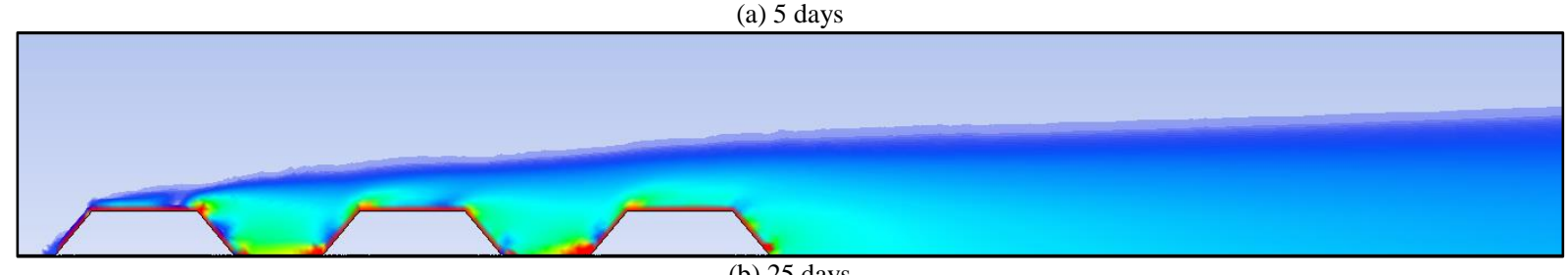

(b) 25 days

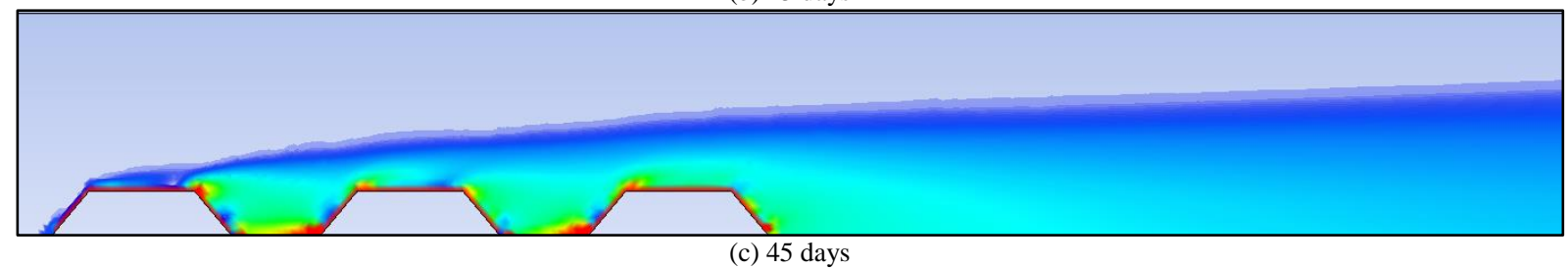

Fig. $13 \mathrm{CO}_{2}$ dispersion (mass fraction) in farfield after various periods

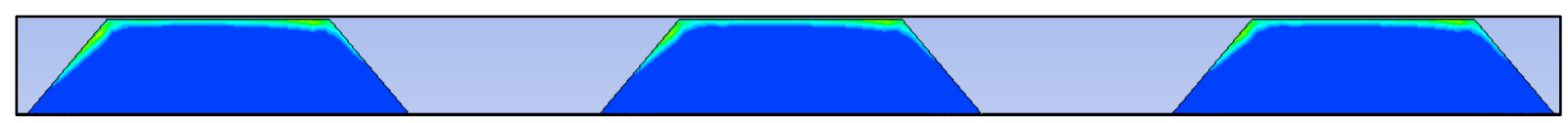

(a) $2 \mathrm{~m} / \mathrm{s}$

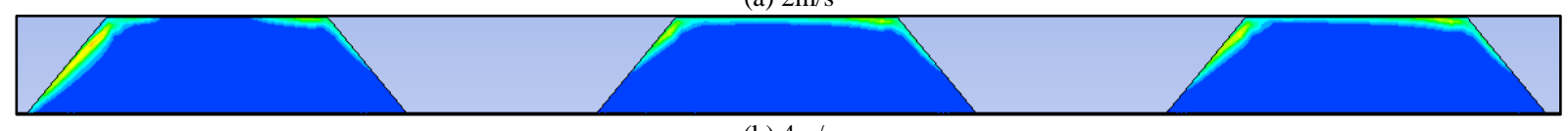

(b) $4 \mathrm{~m} / \mathrm{s}$

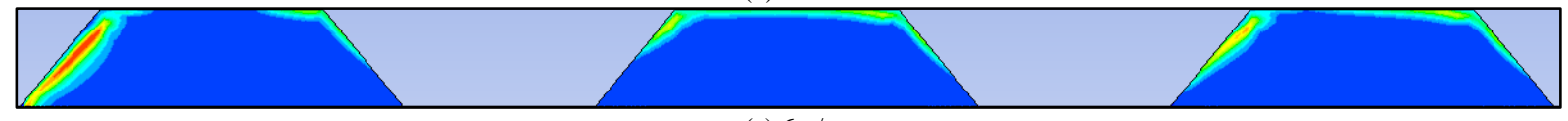

(c) $6 \mathrm{~m} / \mathrm{s}$

Fig. 14 Temperature contours of the three stockpiles of base model with various wind velocities at 30 days 


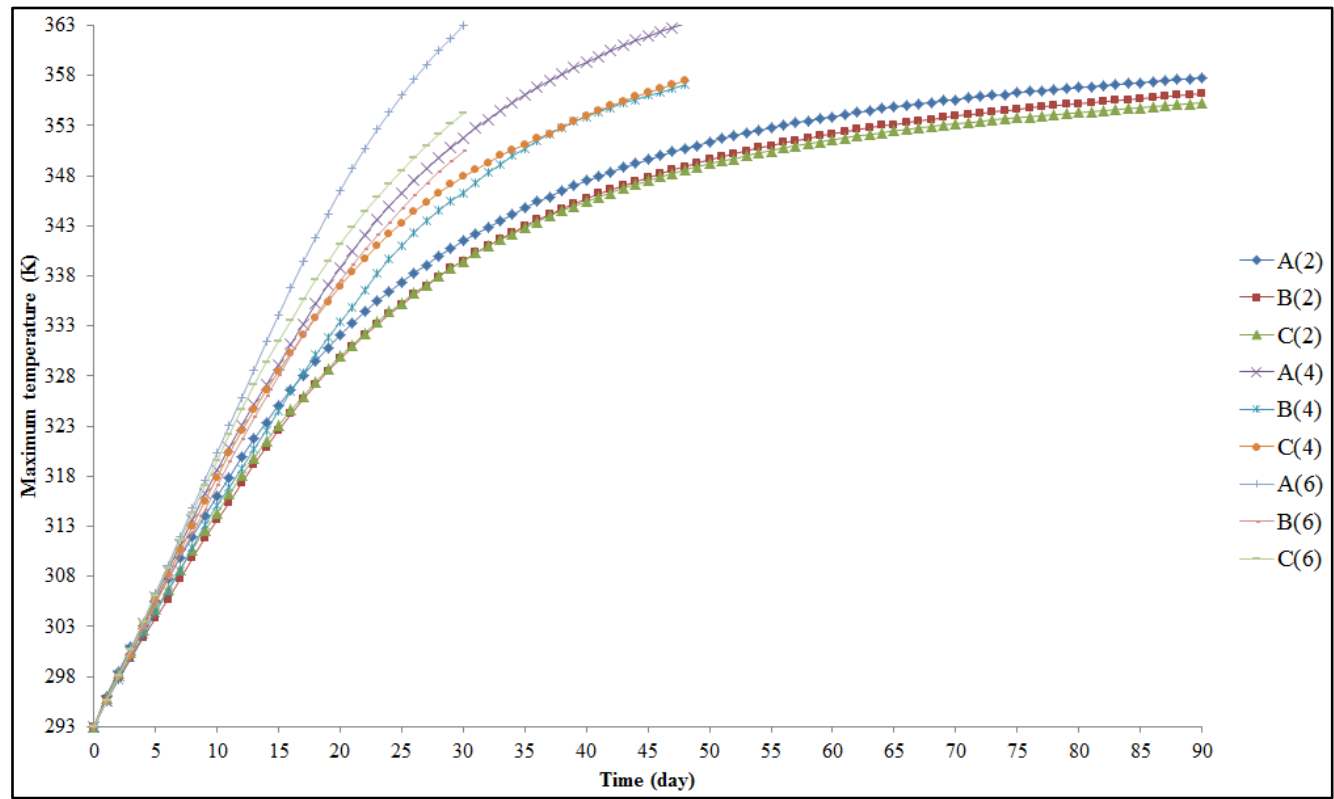

Fig. 15 Maximum temperature rising profiles of base model with various wind velocities *A(2) denotes stockpile-A with $2 \mathrm{~m} / \mathrm{s}$ wind velocity

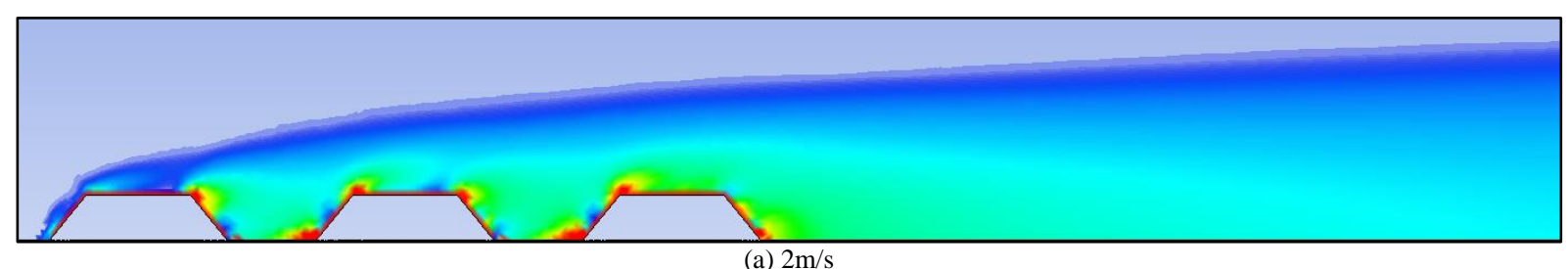

(a) $2 \mathrm{~m} / \mathrm{s}$

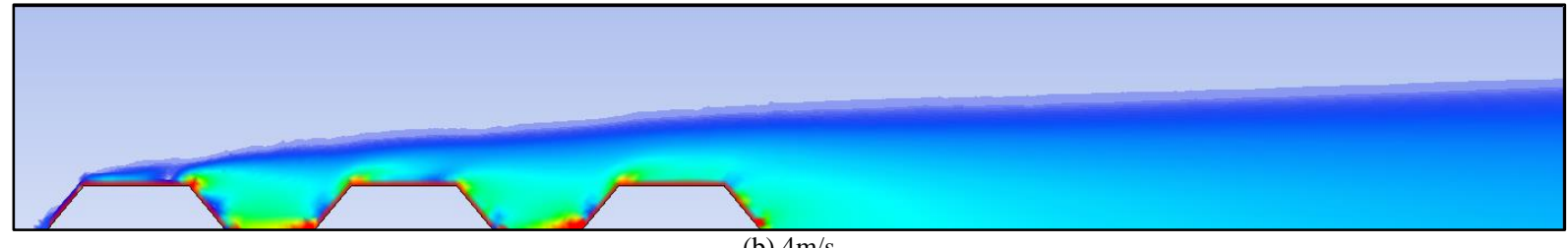

Fig. $16 \mathrm{CO}_{2}$ dispersion (mass fraction) in farfield after 30 days at various wind velocities 


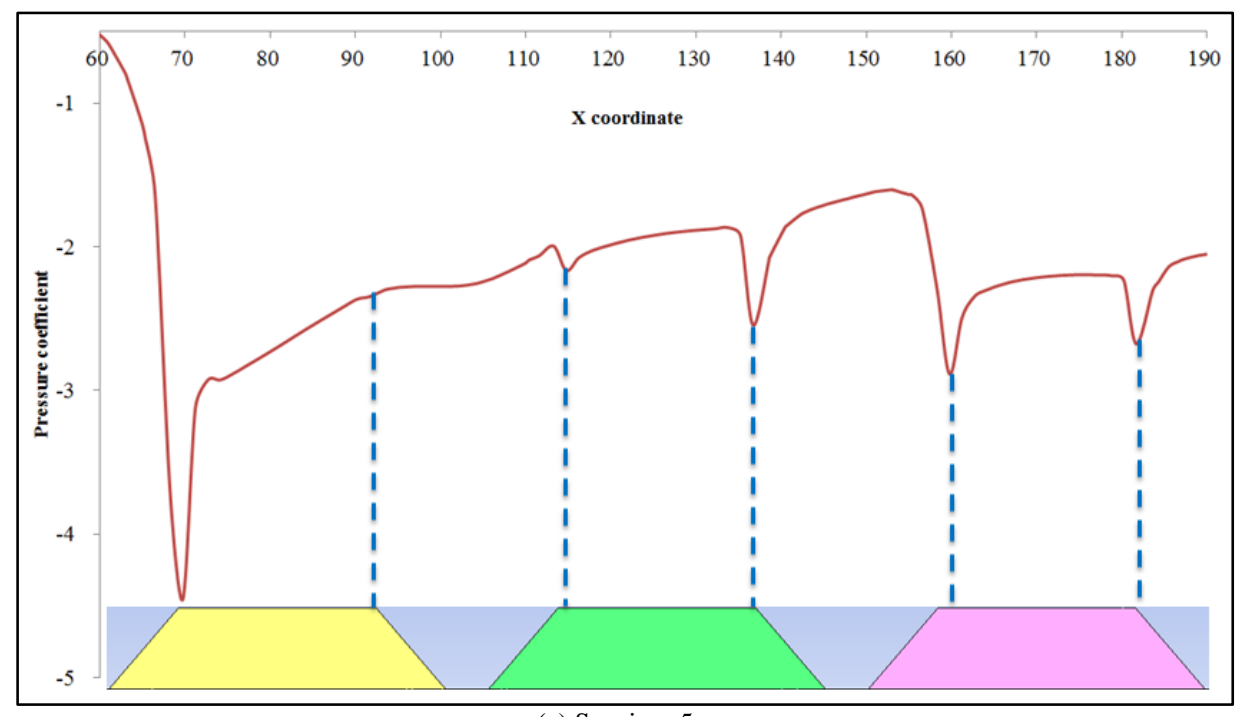

(a) Spacing $=5 \mathrm{~m}$

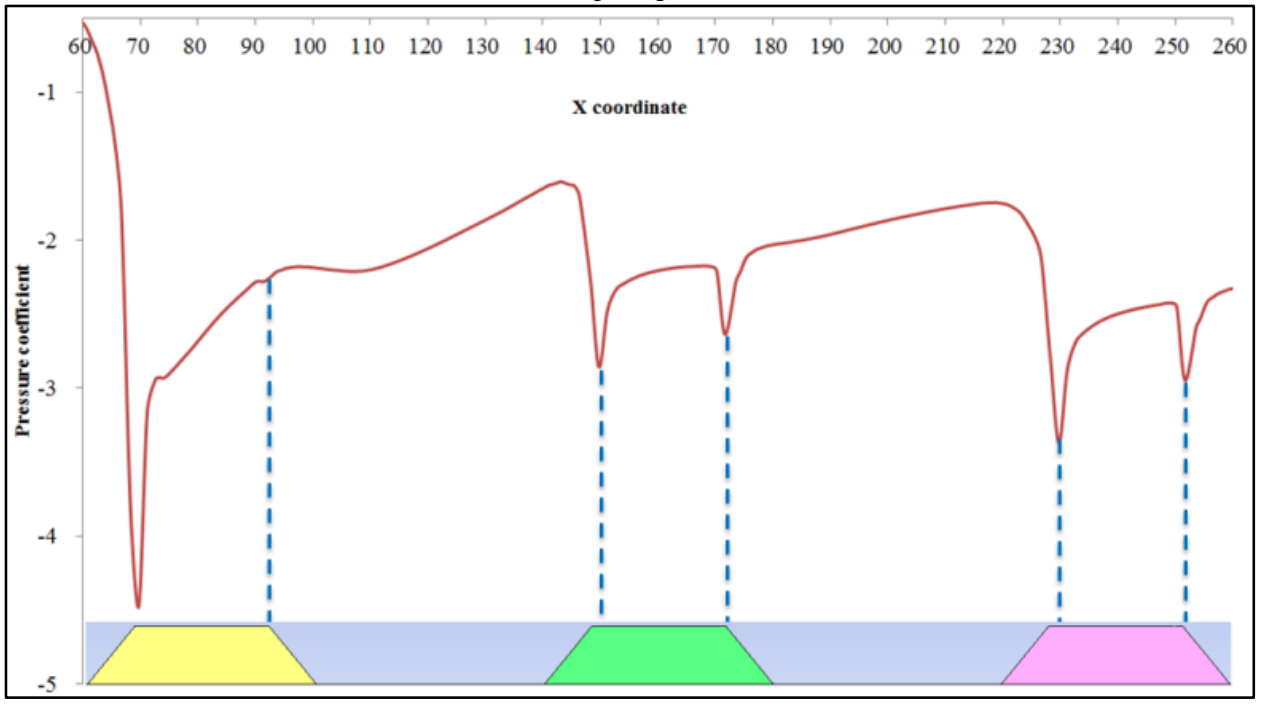

(b) Spacing $=40 \mathrm{~m}$

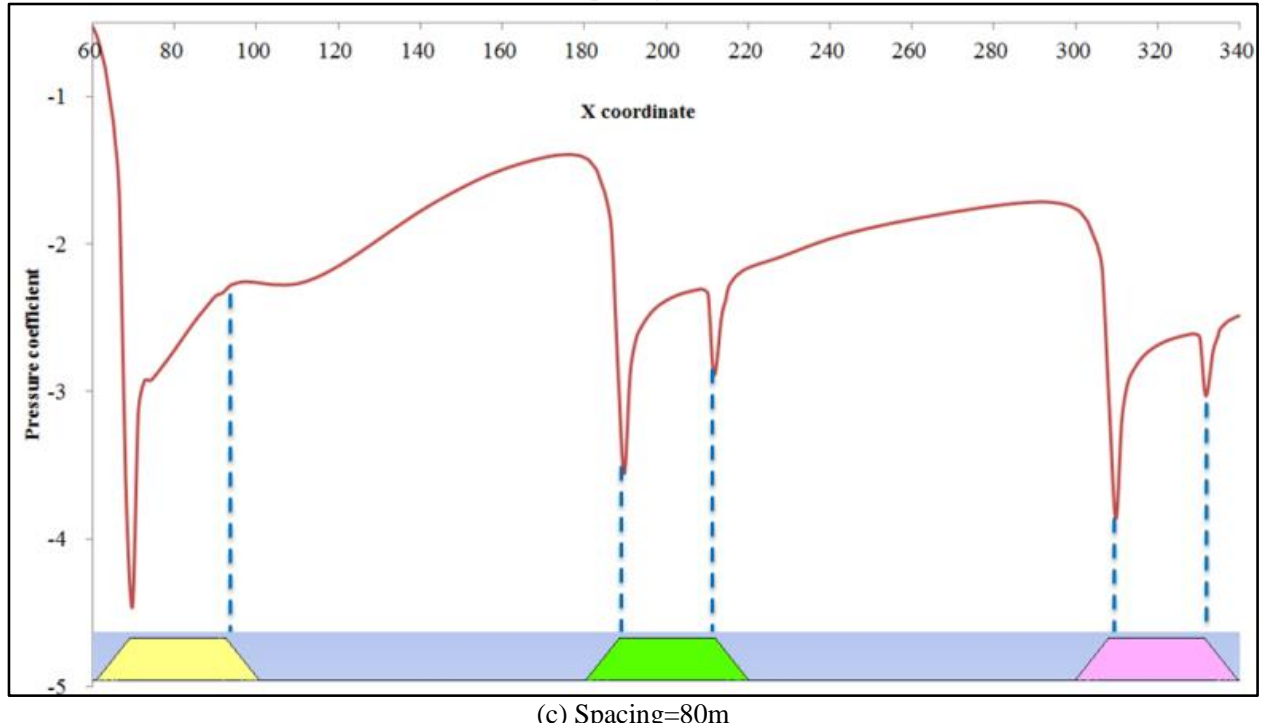

(c) Spacing $=80 \mathrm{~m}$

Fig. 17 Distribution of the pressure coefficient around the three stockpiles with various spacings (wind velocity $=4 \mathrm{~m} / \mathrm{s}$, porosity $=0.2$, height $=10 \mathrm{~m}$, and side slope $=50^{\circ}$ ) 


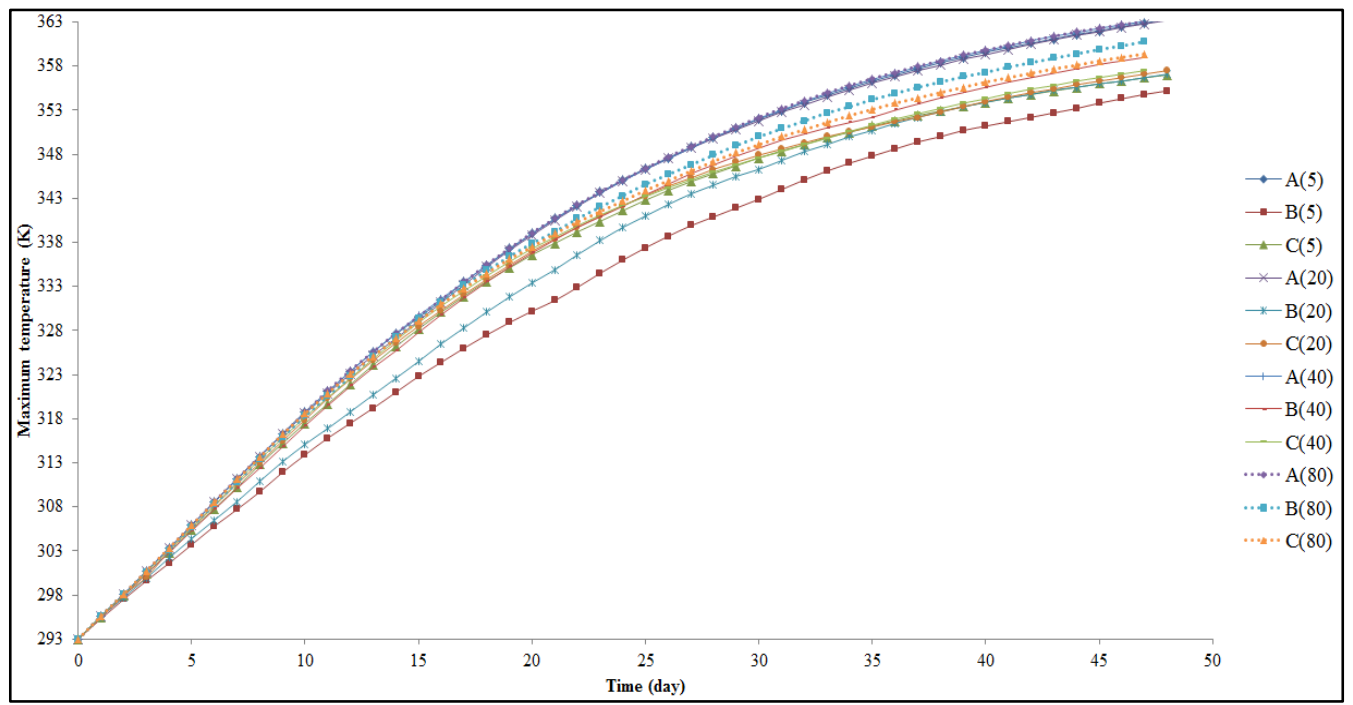

Fig. 18 Maximum temperature rising profiles with various spacings (wind velocity $=4 \mathrm{~m} / \mathrm{s}$, porosity $=0.2$, side slope $=50^{\circ}$, and height $=10 \mathrm{~m}$ )

*A(5) denotes stockpile-A with $5 \mathrm{~m}$ spacing

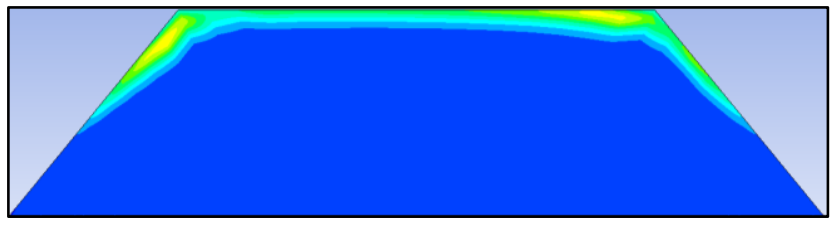

(a) Spacing $=5 \mathrm{~m}$

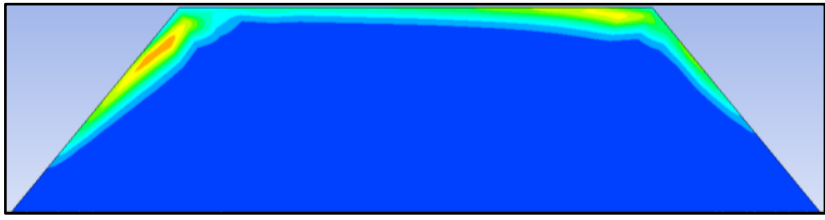

(c) Spacing $=40 \mathrm{~m}$

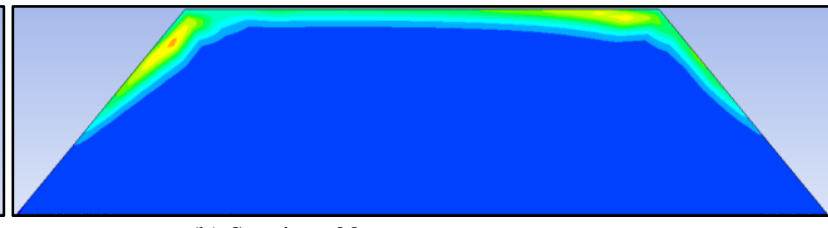

(b) Spacing=20m

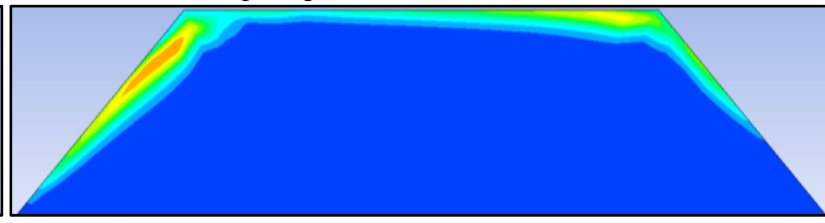

(d) Spacing $=80 \mathrm{~m}$

Fig. 19 Temperature contour of stockpile $B$ with various spacings (time $=30$ days, wind velocity $=4 \mathrm{~m} / \mathrm{s}$, porosity $=0.2$, side slope $=50^{\circ}$, and height $=10 \mathrm{~m}$ )

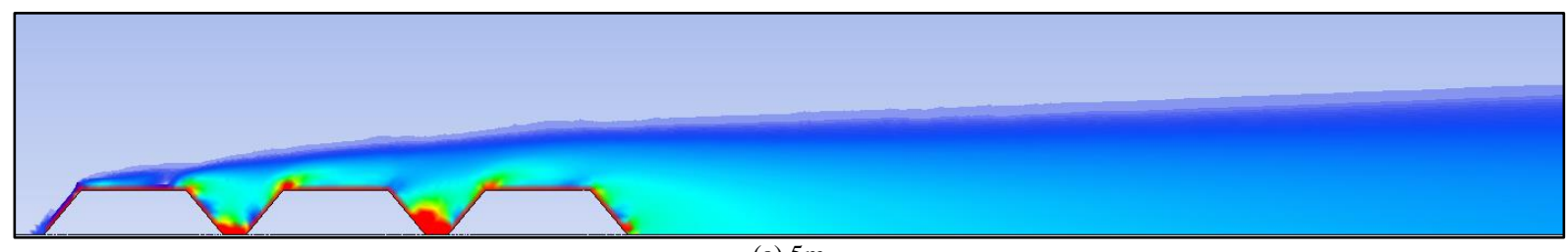

(a) $5 \mathrm{~m}$

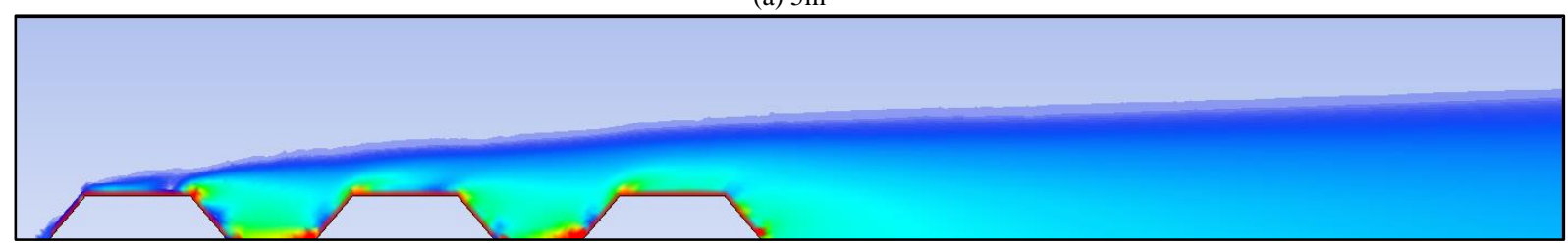

(b) $20 \mathrm{~m}$

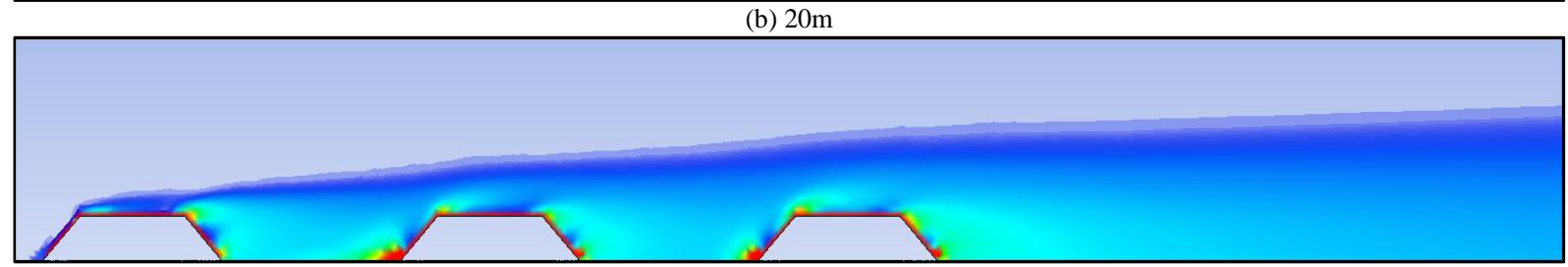

(c) $40 \mathrm{~m}$ 
Fig. $20 \mathrm{CO}_{2}$ dispersion (mass fraction) in farfield with various spacing (wind=4 m/s, time=30 days)

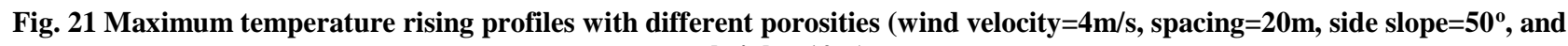
height $=10 \mathrm{~m}$ )

*A(0.3) denotes stockpile-A with 0.3 porosity

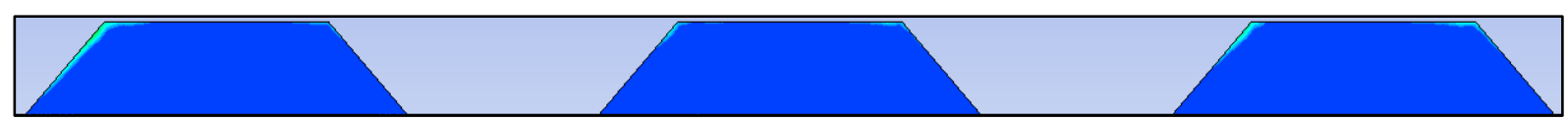

(a) Porosity $=0.1$

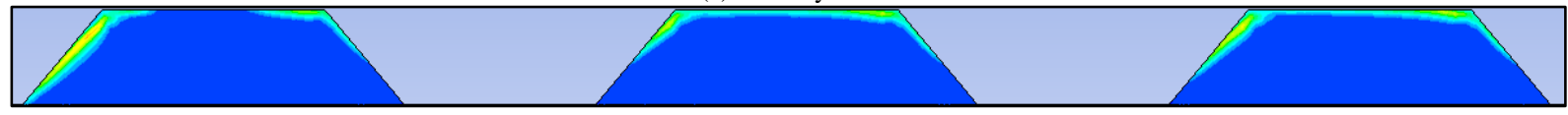

(b) Porosity $=0.2$

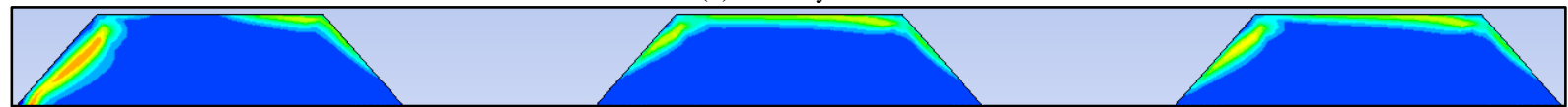

(c) Porosity $=0.3$

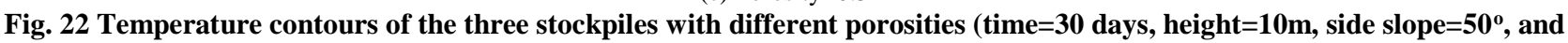
spacing $=20 \mathrm{~m}$ )

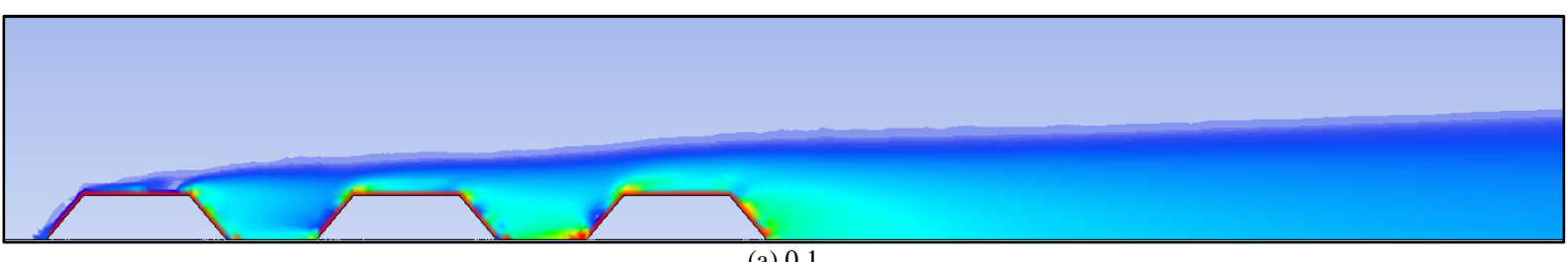

(a) 0.1 

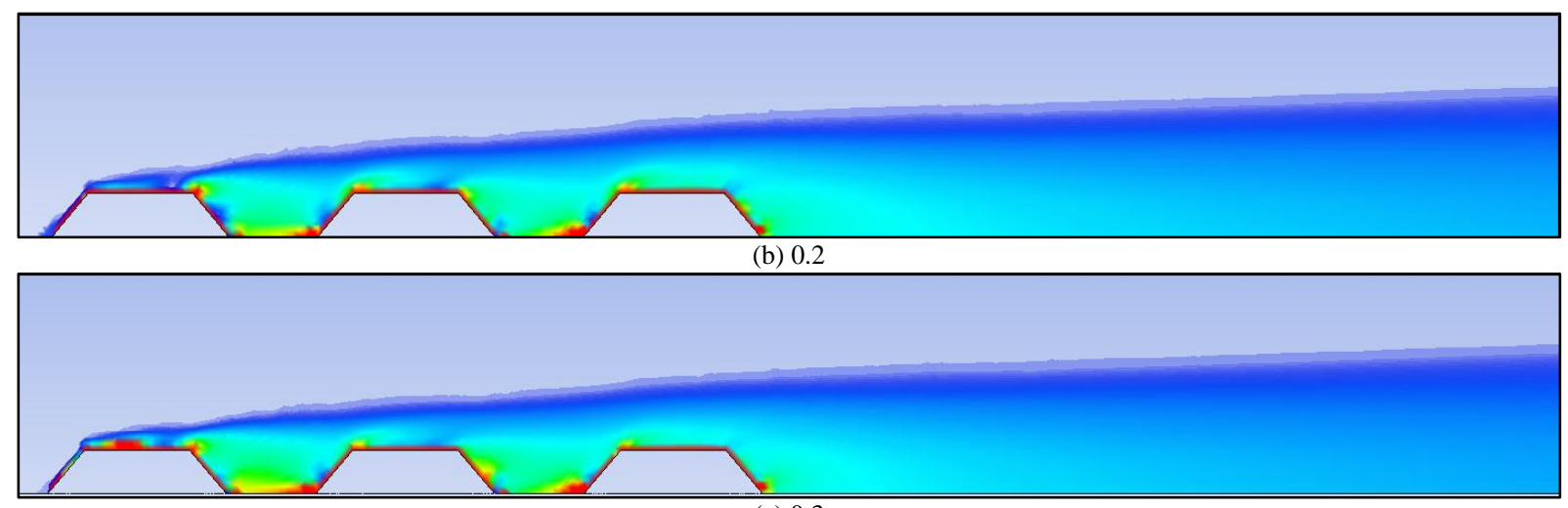

Fig. $23 \mathrm{CO}_{2}$ dispersion (mass fraction) in farfield with various porosities (wind=4 m/s, time=30 days)

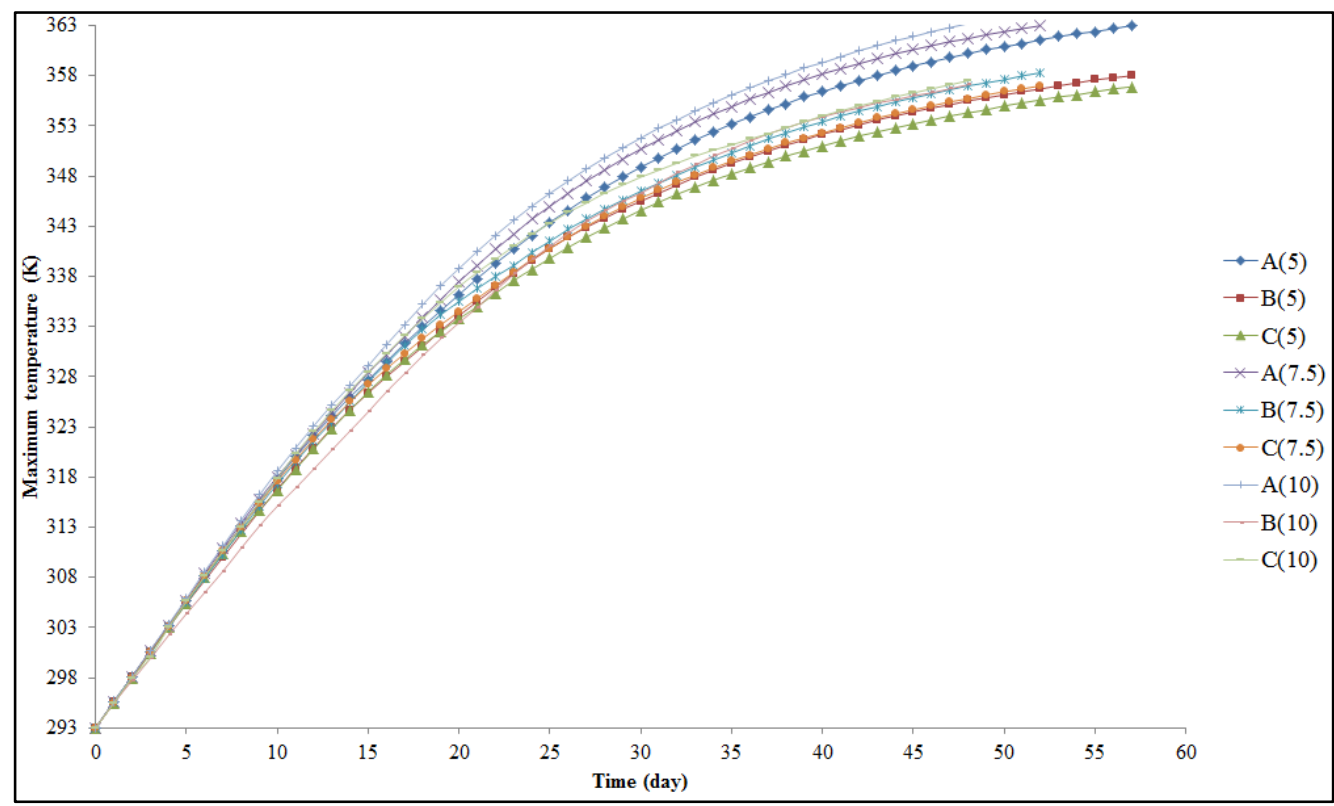

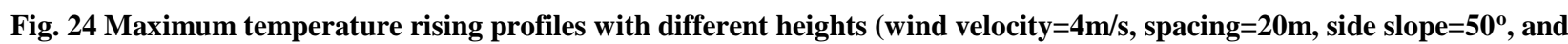
porosity $=0.2$ )

*A(5) denotes stockpile-A with $5 \mathrm{~m}$ height

(a) Height $=5 \mathrm{~m}$

(b) Height $=7.5 \mathrm{~m}$

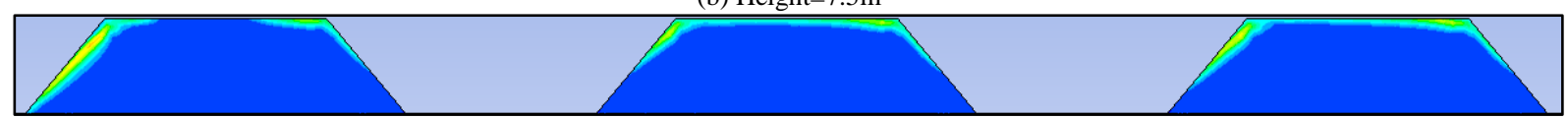

(c) Height $=10 \mathrm{~m}$

Fig. 25 Temperature contours of the three stockpiles with different heights (time $=30$ days, porosity $=0.2$, side slope $=50^{\circ}$, and spacing $=20 \mathrm{~m}$ ) 
Fig. $26 \mathrm{CO}_{2}$ dispersion (mass fraction) in farfield with various heights (wind=4 m/s, time=30 days)

Fig. 27 Maximum temperature rising profiles with different side slopes (wind velocity $=4 \mathrm{~m} / \mathrm{s}$, spacing $=20 \mathrm{~m}$, height= $10 \mathrm{~m}$, and porosity $=\mathbf{0 . 2}$ )

*A(35) denotes stockpile-A with $35^{\circ}$ side slope
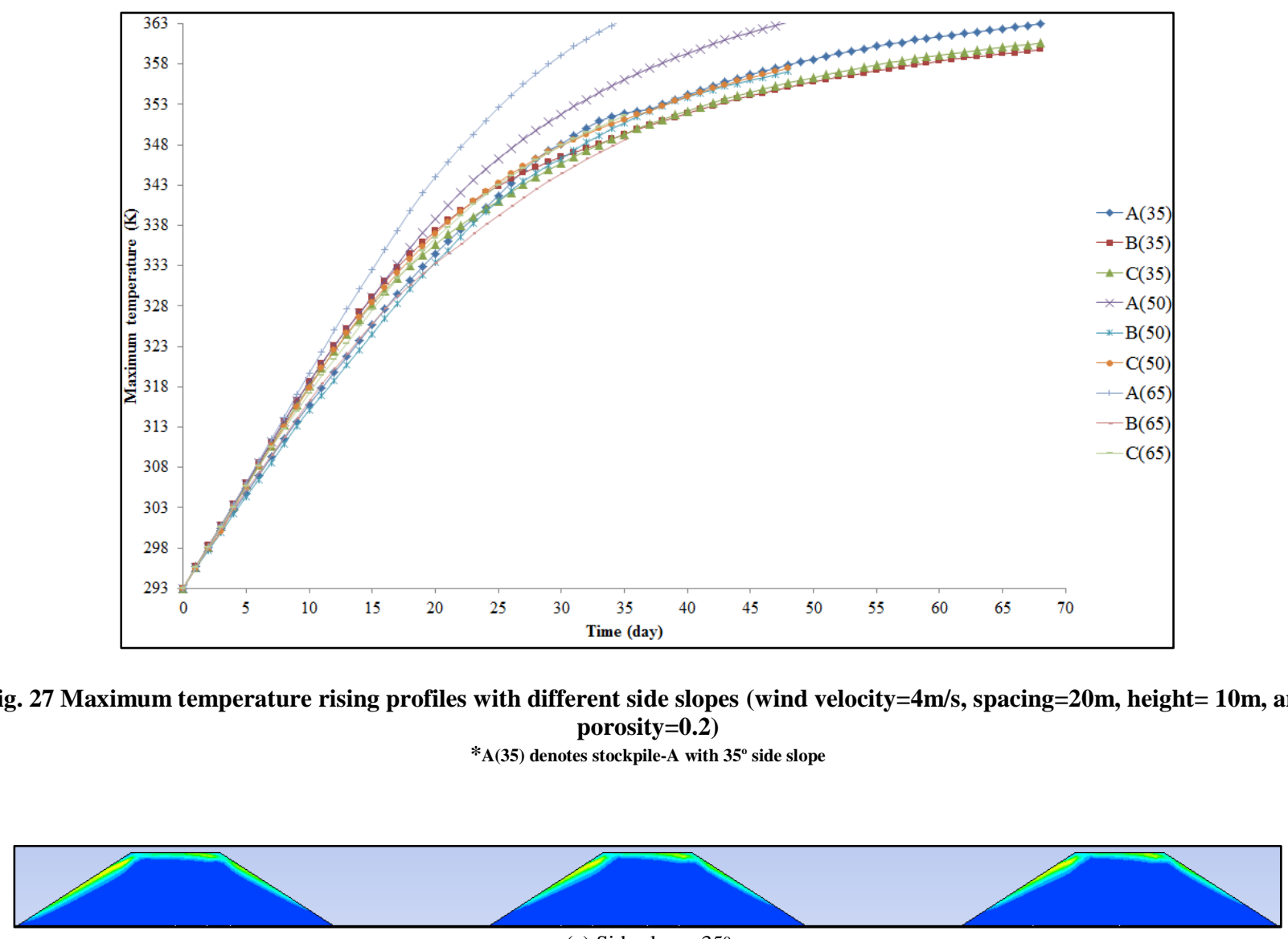

(a) Side slope $=35^{\circ}$

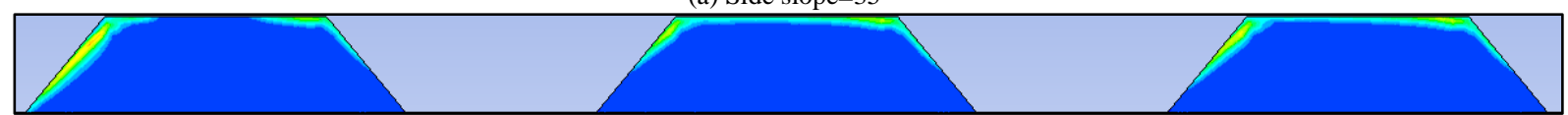

(b) Side slope $=50^{\circ}$

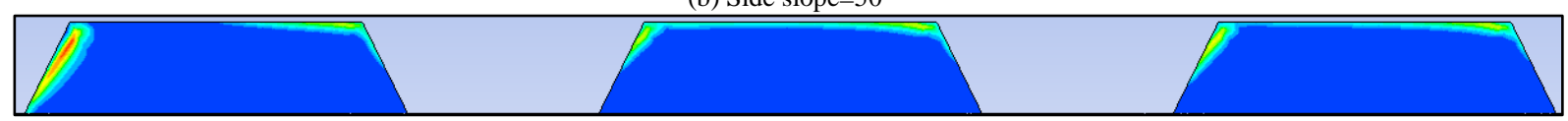

(c) Side slope $=65^{\circ}$ 
Tab. 1 Important input parameters in this model

\begin{tabular}{|c|c|c|}
\hline Specific heat capacity of coal & 1300 & $\mathrm{~J} \mathrm{~kg}^{-1} \mathrm{~K}^{-1}$ \\
\hline Apparent activation energy & 22 & $\mathrm{~kJ} \mathrm{~mol}^{-1}$ \\
\hline Heat of coal oxidation & 300 & $\mathrm{~kJ} \mathrm{~mol}^{-1} \mathrm{O}_{2}$ \\
\hline Apparent order of reaction & 1 & \\
\hline Arrhenius pre-exponential factor & 1.8 & $\mathrm{~s}^{-1}$ \\
\hline Gravitational acceleration & 9.8 & $\mathrm{~m} \mathrm{~s}^{-2}$ \\
\hline Diameter of coal particle & 1 & $\mathrm{~cm}$ \\
\hline Heat transfer coefficient & 3 & $\mathrm{~W} \mathrm{~m} \mathrm{~K}^{-1}$ \\
\hline Density of coal & 1400 & $\mathrm{~kg} \mathrm{~m}^{-3}$ \\
\hline Thermal conductivity of coal & 0.2 & $\mathrm{~W} \mathrm{~m}{ }^{-1} \mathrm{~K}^{-1}$ \\
\hline
\end{tabular}

Tab. 2 Initial and boundary conditions employed in this model

\begin{tabular}{|c|c|c|c|c|c|c|}
\hline \multicolumn{2}{|c|}{ Condition } & Gas flow & $\mathrm{O}_{2}$ transport & $\mathrm{CO}_{2}$ transport & $\mathrm{CO}$ transport & Temperature \\
\hline \multicolumn{2}{|c|}{ Initial conditions } & $\mathrm{V}=0(\mathrm{~m} / \mathrm{s})$ & $\mathrm{C}_{\mathrm{o}}=0$ & $\mathrm{C}_{\mathrm{i}}=0$ & $C_{j}=0$ & $293 \mathrm{~K}$ \\
\hline \multirow{5}{*}{$\begin{array}{l}\text { Boundary } \\
\text { conditions }\end{array}$} & Inlet $(x=0)$ & $\begin{array}{c}\text { Velocity } \\
\text { inlet }\end{array}$ & $\begin{array}{c}\mathrm{C}_{0}=0.23 \text { (mass } \\
\text { fraction) }\end{array}$ & $\mathrm{C}_{\mathrm{i}}=0$ & $C_{j}=0$ & $293 \mathrm{~K}$ \\
\hline & $\begin{array}{l}\text { Upper wall } \\
(\mathrm{y}=50)\end{array}$ & No flux & No flux & No flux & No flux & Adiabatic \\
\hline & $\begin{array}{l}\text { Lower wall } \\
\quad(y=0)\end{array}$ & No flux & No flux & No flux & No flux & Adiabatic \\
\hline & Interface & $\begin{array}{c}\text { Convection } \\
\text { flux }\end{array}$ & $\begin{array}{l}\text { Diffusion and } \\
\text { convection flux }\end{array}$ & $\begin{array}{c}\text { Diffusion and } \\
\text { convection flux }\end{array}$ & $\begin{array}{c}\text { Diffusion and } \\
\text { convection flux }\end{array}$ & $\begin{array}{c}\text { Conduction and } \\
\text { convection }\end{array}$ \\
\hline & $\begin{array}{c}\text { Outlet } \\
(\mathrm{x}=400)\end{array}$ & Outflow & - & - & 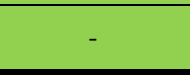 & 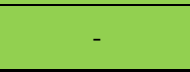 \\
\hline
\end{tabular}

